ARTICLE

DOI: $10.1038 / \mathrm{s} 41467-018-03014-4$

\title{
Identification of the fungal ligand triggering cytotoxic PRR-mediated NK cell killing of Cryptococcus and Candida
}

Shu Shun Li1,2, Henry Ogbomo ${ }^{1,2}$, Michael K. Mansour ${ }^{3}$, Richard F. Xiang ${ }^{1,2}$, Lian Szabo4 ${ }^{4}$ Fay Munro ${ }^{5}$, Priyanka Mukherjee ${ }^{5}$, Roy A. Mariuzza ${ }^{6}$, Matthias Amrein ${ }^{5}$, Jatin M. Vyas (1) ${ }^{3}$, Stephen M. Robbins ${ }^{7,8} \&$ Christopher H. Mody (1) 1,2,4

Natural killer (NK) cells use the activating receptor NKp30 as a microbial pattern-recognition receptor to recognize, activate cytolytic pathways, and directly kill the fungi Cryptococcus neoformans and Candida albicans. However, the fungal pathogen-associated molecular pattern (PAMP) that triggers NKp30-mediated killing remains to be identified. Here we show that $\beta$ 1,3-glucan, a component of the fungal cell wall, binds to NKp30. We further demonstrate that $\beta-1,3-$ glucan stimulates granule convergence and polarization, as shown by live cell imaging. Through Src Family Kinase signaling, $\beta-1,3$-glucan increases expression and clustering of NKp3O at the microbial and NK cell synapse to induce perforin release for fungal cytotoxicity. Rather than blocking the interaction between fungi and NK cells, soluble $\beta-1,3-$ glucan enhances fungal killing and restores defective cryptococcal killing by NK cells from HIVpositive individuals, implicating $\beta-1,3-$ glucan to be both an activating ligand and a soluble PAMP that shapes NK cell host immunity.

\footnotetext{
${ }^{1}$ Department of Microbiology, Immunology and Infectious Diseases, University of Calgary, Calgary T2N 4N1, Canada. ${ }^{2}$ The Calvin, Phoebe and Joan Snyder Institute for Chronic Diseases, University of Calgary, Calgary T2N 4N1, Canada. ${ }^{3}$ Department of Medicine Division of Infectious Diseases, Massachusetts General Hospital, Boston, MA 02114, USA. ${ }^{4}$ Department of Medicine, University of Calgary, Calgary T2N 4N1, Canada. ${ }^{5}$ Department of Cell Biology and Anatomy, University of Calgary, Calgary T2N 4N1, Canada. ${ }^{6}$ Department of Cell Biology \& Molecular Genetics, University of Maryland, College Park, MD 20742, USA. ${ }^{7}$ Department of Biochemistry and Molecular Biology, University of Calgary, Calgary T2N 4N1, Canada. ${ }^{8}$ Southern Alberta Cancer Research Institute, University of Calgary, Calgary T2N 4N1, Canada. Shu Shun Li and Henry Ogbomo contributed equally to this work Correspondence and requests for materials should be addressed to C.H.M. (email: cmody@ucalgary.ca)
} 
nvasive fungal infections are both widespread and increasing in frequency, particularly in immunocompromised individuals ${ }^{1,2}$. Cryptococcus neoformans (C. neoformans) causes life-threatening meningitis and pneumonia in AIDS and other immunosuppressed patients, while Candida albicans (C. albicans) produces a devastating mycosis in post-surgical and critically ill patients ${ }^{3,4}$. Understanding host defense for developing more effective treatment or control of fungal invasive infections remains a major challenge.

Innate immune host defense is evolutionally conserved, and has an essential function in immunity against microbial infections. Natural killer (NK) cells are innate cytotoxic cells that directly recognize and kill C. neoformans and C. albicans. In mice, NK cells are required for optimal clearance of $C$. neoformans and C. albicans $^{5-9}$. In humans, NK cells bind to C. neoformans and mediate killing ${ }^{10,11}$. By contrast, NK cell function is defective in patients with cryptococcal meningitis ${ }^{12}$, and NK cells from HIVinfected patients are unable to kill C. neoformans ${ }^{13}$. NK cells depleted of the critical effector molecule, perforin, could be seen in contact with Cryptococcus within the brain of a patient that succumbed to the infection ${ }^{14}$. Previously, we demonstrated that the NK cell receptor, NKp30, is the pattern-recognition receptor (PRR) recognizing C. neoformans and C. albicans that triggers activation of PI3K and Erk 1/2, perforin release, and fungal cytotoxicity ${ }^{15}$.

PRRs are proteins expressed by cells of the immune system that recognize pathogen-associated molecular patterns (PAMPs) as danger signals. PRR were previously organized into two categories. Phagocytic PRRs, such as Dectin-1, MARCO, scavenger receptor A, and mannose receptors, are expressed by macrophages, dendritic cells, monocytes, and neutrophils, and activate phagocytosis upon binding of a microbial PAMP ${ }^{16-19}$. Signaling PRR are transmembrane or cytoplasmic receptors that stimulate gene transcription of pro-inflammatory cytokines, type I interferons, chemokines, antimicrobial peptides, and costimulatory molecules in a wide variety of immune and non-immune cells. Signaling PPRs include extracellular Toll-like receptors, C-type lectin receptors, intracellular nucleotide-binding oligomerization domain-like receptors (NLR), and retinoic acid inducible gene Ilike helicase receptors (RLR) ${ }^{20}$. In addition to these categories, a new class of PRR has been described that includes NK cellactivating receptors, $\mathrm{NKp} 30, \mathrm{NKp} 46$, and CD56 that bind to fungi and parasites to induce mobilization and release of cytotoxic granules that kill the pathogen ${ }^{15,21-23}$. NKp30, NKp46, and CD56 are all members of the immunoglobulin-like transmembrane receptor family that use ITAM-containing adaptor proteins to signal. Studies demonstrating direct binding to fungal and parasitic PAMPs suggest that Ig-like family members that activate NK cells for microbial killing be added to the PRR families forming a cytotoxic PRR subfamily. Although a PAMP for NKp46 has been identified $^{21}$, the microbial PAMP for the cytotoxic PRR NKp30 remains to be identified.

PAMPs often serve as an essential function in the pathogen and are often shared among entire classes of microbes. Molecules expressing PAMPs are either structural determinants or required for virulence ${ }^{24}$. The structure of $C$. neoformans consists of a unique polysaccharide capsule that surrounds the organism ${ }^{25}$. Beneath the capsule is the cell wall and membrane. The cell wall consists of a complex organization of polysaccharides, with smaller amounts of proteins, lipids, and pigments, that are directly exposed in C. albicans and acapsular C. neoformans, and to a lesser extent by encapsulated Cryptococcus ${ }^{26,27}$. Polysaccharides constitute nearly $80 \%$ of the fungal dry weight and include glucuronoxylomannan and galactoxylomannan in the capsule, and glucans, chitin, and chitosan in the cell wall. NKp30 mediates NK cell killing of both acapsular Cryptococcus and C. albicans as well as encapsulated C. neoformans; therefore, the potential ligand for NKp30 is not a component of the cryptococcal capsule. Additionally, Cryptococcus (phyla Basidiomycota) is separated from C. albicans (phyla Ascomycota) by 400 million years of evolution ${ }^{28}$, suggesting that the ligand for NKp30 is essential and preserved among widely divergent phyla. Since glucans are major structural components of fungal cell walls, our focus was narrowed to a limited subset of $\beta$-glucans that were the most likely candidates for the NKp30 ligand.

We used a variety of approaches including antibody detection and atomic force spectroscopy to demonstrate that soluble and immobilized $\beta$-1,3-glucan binds NKp30. We found that $\beta$-1,3glucan induces Src family kinase signal transduction, synapse formation, and cytotoxic granule trafficking as seen by live cell imaging. $\beta$-1,3-glucan is necessary for killing, using fungi treated with an echinocandin as a loss-of-function approach. Surprisingly, soluble $\beta$-1,3-glucan enhances receptor and effector molecule expression and enhances killing in NK cells from healthy as well as HIV-infected patients with defective antifungal activity.

\section{Results}

$\boldsymbol{\beta}-1,3$-glucan binds to NK cells. Since the same receptor, NKp30, mediates NK cell recognition and killing of $C$. neoformans and $C$. albicans, it was likely that the fungal ligand is shared between these widely divergent phyla. We reasoned that an essential structural molecule was the likely candidate. As such, $\alpha$-glucans and $\beta$-glucans are the major structural components of fungal cell walls. Since the former is not found in the cell wall of C. albicans $^{29}, \alpha$-glucan was excluded from our list of candidates as a ligand for NKp30. The latter includes $\beta$-1,3-glucan, $\beta$-1,4-glucan, $\beta$-1,6-glucan, or mixes of $\beta$-1,3-/ $\beta-1,4$ - or $\beta$-1,6-glucan. However, C. albicans and C. neoformans share only $\beta-1,3$-glucan and $\beta-1,6-$ glucan ${ }^{29,30}$, which narrowed our focus.

Experiments were performed to examine whether $\beta$-glucans could bind to YT cells, an NK cell line that kills Cryptococcus and Candida ${ }^{15}$. YT cells were incubated with a preparation of the cryptococcal cell wall/membrane $(\mathrm{CCW} / \mathrm{M})^{31}$. Antibody labeling and flow cytometry demonstrated $\beta$-1,3-glucan on the surface of YT cells (Fig. 1a). To test if soluble $\beta$-glucans bound to YT cells, the cells were incubated with laminarin (derived from Laminaria digitata), consisting of $\beta-1,3$-glucan with $\beta-1,6$ branches (ratio $\sim 3: 1)^{32}$. $\beta-1,3$-glucan was detected on YT cells (Fig. 1b, left panel), but not on untreated YT cells (Fig. 1b, right panel). Since $\beta$ glucan from $L$. digitata and Saccharomyces cerevisiae ( $S$. cerevisiae) is a mixture of $\beta-1,3$ and $\beta-1,6$-glucans, experiments were performed to determine whether the binding was due to $\beta$ 1,3-glucan. For this purpose, the experiment was repeated with soluble laminarihexaose produced from curdlan, which comprises only $\beta$-1,3-glucan or pustulan, comprising only $\beta$-1,6-glucan. We found that laminarihexaose bound to YT cells (Fig. 1c), while pustulan did not (Fig. 1d), indicating that $\beta-1,3$-glucan is sufficient for binding to YT cells.

及-1,3-glucan binds to recombinant NKp30. To determine whether the immobilized $\beta$-glucan bound to NKp30, polystyrene beads were conjugated with or without $\beta$-glucan derived from $S$. cerevisiae (Supplementary Fig. $1 \mathrm{~A}-\mathrm{B})^{33}$, which consists of $\beta-1,3$ glucan with $\beta-1,6$ branches (ratio $\sim 5: 1)^{29}$. Binding of a recombinant Fc-NKp30 fusion protein to $\beta$-glucan-conjugated beads ( $\beta$ $1,3-\mathrm{GB})$ was revealed by flow cytometry using a polyclonal antiNKp30 antibody (Fig. 1e).

$1 \mathrm{C} 01$ is a monoclonal antibody $(\mathrm{mAb})$ that binds to NKp30 and blocks the recognition of fungi ${ }^{15}$. We questioned whether this antibody would block the interaction of NKp30 with its potential binding partner, $\beta$-1,3-glucan. To test this, NKp30-Fc was 
incubated with or without $1 \mathrm{C} 01$, and the mixture was incubated with $\beta-1,3-\mathrm{GB}$. We were unable to detect the presence of NKp30 on $\beta-1,3-\mathrm{GB}$ using a polyclonal anti-NKp30 antibody, suggesting that mAb $1 \mathrm{C} 01$ blocked the binding of NKp30-Fc to $\beta-1,3-\mathrm{GB}$ (Fig. 1f). We considered the possibility that $1 \mathrm{C} 01$ might block the binding site for polyclonal anti-NKp30; however, this was not the case as YT cells demonstrated similar labeling with polyclonal anti-NKp30 with or without pre-incubation with 1C01 (Supplementary Fig. 1C). This reveals that $1 \mathrm{C} 01$ was bound to, or resulted in, steric hindrance of the $\beta$-1,3-glucan binding site on NKp30.

Both C. neoformans and $C$. albicans express $\beta$-1,3-glucan in their cell wall. To determine whether the ectodomain of NKp30 could bind to these two microorganisms, we incubated recombinant NKp30 ectodomain with C. neoformans or C. albicans, and labeled them with anti-NKp30 Ab. NKp30 was detected by flow cytometric analysis (Fig. 1g) consistent with NKp30 binding to $\beta$-1,3-glucan in these two fungi.

To determine whether $\beta$-1,3-glucan could immunoprecipitate NKp30 from YT cells, protein G beads were conjugated with an $\mathrm{mAb}$ against $\beta$-1,3-glucan. The beads were incubated with a YT cell lysate that had been incubated with $\beta$-1,3-glucan (laminarin). Western blotting showed that NKp30 was immunoprecipitated by $\beta$-1,3-glucan bound to anti- $\beta$-1,3-glucan mAb-coated beads (Fig. $1 \mathrm{~h}, 2^{\text {nd }}$ lane from left), providing additional evidence that NKp30 is the binding partner for $\beta-1,3$-glucan. a

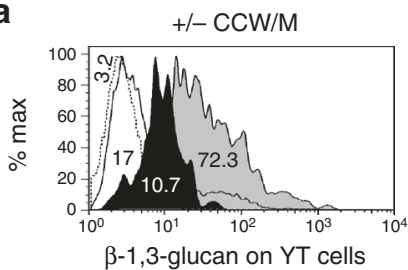

$\square$ YT alone $\quad \square-\mathrm{CCW} / \mathrm{M}$ (mAb)

+ CCW/M (iso) $\square+$ CCW/M (mAb)

C

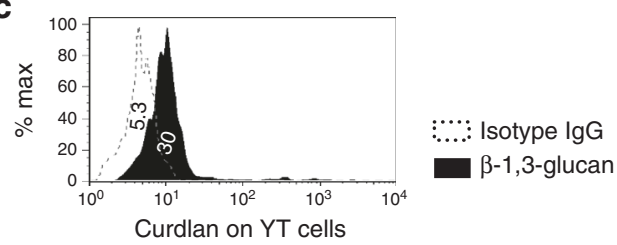

e
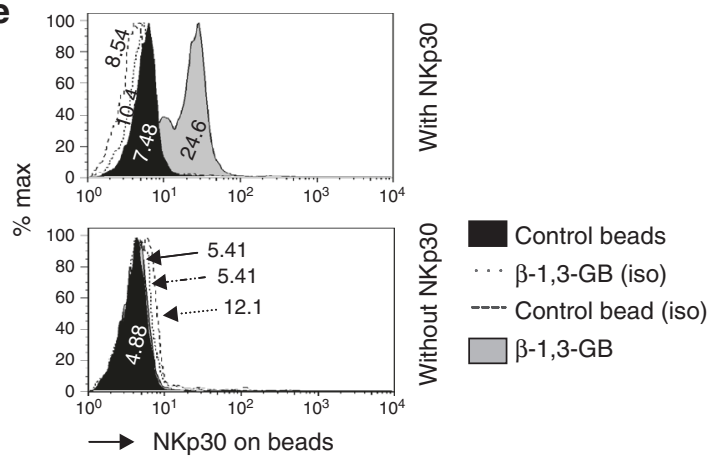

9
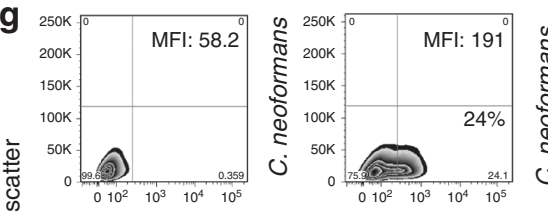

के 250
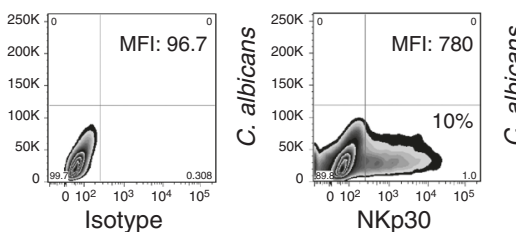

h

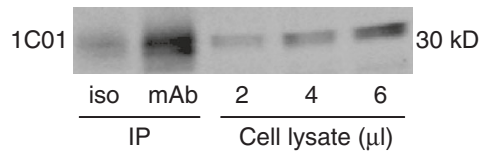

b
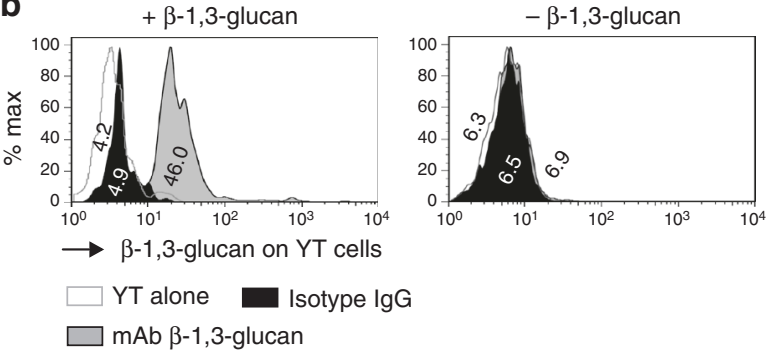

d $10^{4}$

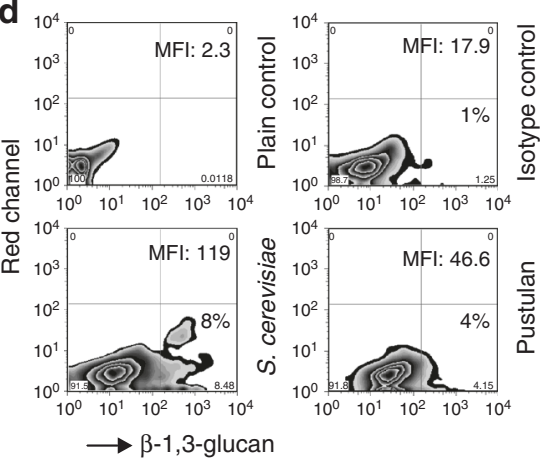

$\longrightarrow \beta$-1,3-glucan

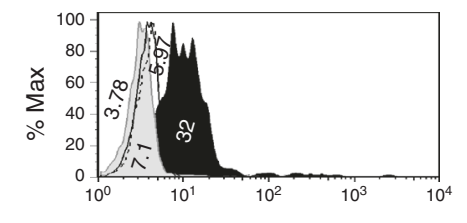

NKp30 on $\beta-1,3-G B$

$\square$ Plain control (MFI: 3.78 )

:....: Isotype control, MFI: 5.97)

- Anti-NKp30 Ab (- 1C01) (MFI: 32)

Anti-NKp30 Ab (+ 1C01) (MFI: 7.1)

i
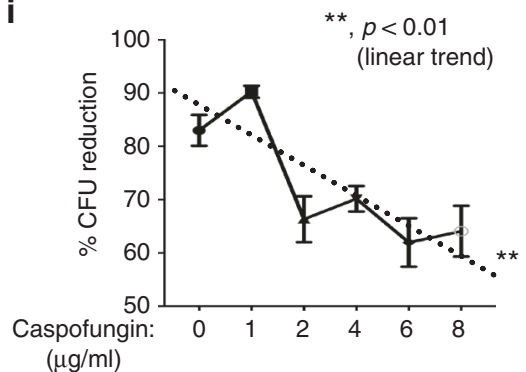
及-1,3-glucan is required for NK killing of Cryptococcus. To explore whether $\beta$-1,3-glucan was required for NK cell killing of Cryptococcus, we took advantage of the ability of caspofungin to interrupt $\beta$-1,3-glucan synthesis, despite the relative resistance of C. neoformans to this agent ${ }^{34,35}$. C. neoformans (B3501) was treated with various concentrations of caspofungin and NK cell killing was examined. As expected, caspofungin reduced the percentage of $\beta$-1,3-glucan-positive C. neoformans (strain B3501, Supplementary Fig. 2a) and expression of $\beta$-1,3-glucan (Supplementary Fig. 2B). While there was a modest effect on the growth of C. neoformans (Supplementary Fig. 2C), YT cell anticryptococcal activity was reduced after treatment with caspofungin in a dose-dependent manner (Fig. 1i and Supplementary Fig. 2C, triangles) revealing that $\beta$-1,3-glucan was required for $\mathrm{NK}$ cell host defense against Cryptococcus.

$\beta-1,3$-glucan enhances NK cell killing in HIV patients. Since $\beta$ 1,3-glucan bound to NKp30 and was required for NK cell killing, we speculated that free $\beta$-1,3-glucan would bind and block NKp30 receptors on NK cells and abrogate NK killing. To test this hypothesis, we pretreated YT cells with soluble laminarin before the addition of $C$. neoformans. To our surprise, $\beta-1,3-$ glucan did not block, but instead, elicited dose-dependent enhanced YT cell killing of C. neoformans (Fig. 2a). Having previously shown that NKp30 mediates NK cell killing of $C$. albicans $^{15}$, the experiments were performed to determine whether $\beta$-1,3-glucan would also enhance the killing of C. albicans. We found that $\beta$-1,3-glucan stimulated a dose-dependent increase in YT cell killing of $C$. albicans (Fig. 2b). $\beta$-1,3-glucan from other sources similarly enhanced YT cell killing of $C$. neoformans (Supplementary Fig. 3A-B). Notably, $\beta$-1,3-glucan showed no impact on YT cell viability ( $>95 \%$ ) or growth of Cryptococcus alone (Supplementary Fig. 3C). The $\beta$-1,3-glucans used in this study were derived from several sources including $L$. digitata and S. cerevisiae that contain glucans with $\beta-1,3$ - and $\beta-1,6$ - branches. To examine whether $\beta$-1,6-glucan contributed to the enhanced killing, YT cells were incubated with pustulan, which contains only $\beta$-1,6-glucans. Treatment of YT cells with $\beta$-1,6-glucans failed to enhance killing (Supplementary Fig. 3D).

We also tested the ability of $\beta$-1,3-glucan to enhance cytotoxicity of primary NK cells against Cryptococcus. Primary NK cells from healthy individuals were pretreated with $\beta-1,3-$ glucan as described above, and similarly, killing by primary NK cells was enhanced (Fig. 2c). Since NK cells from HIV-infected patients have defective killing of $C$. neoformans and this defect can be restored by using interleukin-12 15,36 , we wondered if $\beta$ - 1,3-glucan might have a similar effect. $\beta$-1,3-glucan enhanced killing of Cryptococcus by NK cells from HIV-infected individuals (Fig. 2d).

及-1,3-glucan binds NK cells as shown by live cell AFM. To explore the mechanism by which $\beta$-1,3-glucan enhanced and restored defective NK cell cytotoxicity, we characterized NK cell binding to $\beta$-1,3-glucan using an atomic force microscope to perform single-cell force spectroscopy (SCFS). This method has the advantage of being able to measure the binding force of cell surface receptors with their ligands on live cells during controlled physiologic conditions (temperature, $\mathrm{pH}$, etc.) ${ }^{37}$. A single YT cell was fixed to a cantilever tip, and purified $\beta$-1,3-glucan, mannan $(\alpha-1,2, \alpha-1,3-$, and $\alpha-1,6-\mathrm{D}-\mathrm{mannose}$ in fungi), or pustulan was conjugated to the glass surface (coating in Supplementary Fig. 4A and schematic drawing for binding in Supplementary Fig. 4B). Force-distance (F-D) curves from a single YT cell detaching from $\beta$-1,3-glucan required $2000-3000 \mathrm{pN}$ (Fig. 3a, third panel from left). Heat-killed $S$. cerevisiae, which was the source of $\beta-1,3$ glucan used in some experiments, were adhered to glass to make a nearly confluent single cell layer as illustrated (Supplementary Fig. 4C, F). A representative F-D curve showed the force used to detach a single YT cell from $S$. cerevisiae was greater than from control $(470 \pm 141$ vs. $45 \pm 11 \mathrm{pN})$, but less than that from purified $\beta$-1,3-glucan (Fig. 3a, the fourth panel from left), suggesting that $\beta$-1,3-glucan could account for the binding force between YT cells and Saccharomyces cells. The accumulated retraction forces from multiple touches (Fig. 3b, left panel) and mean retraction forces (Fig. $3 \mathrm{~b}$, right panel) to the $\beta$-1,3-glucan-conjugated surface were significantly higher than to the controls ( $p<0.01$, by one-way ANOVA). The binding forces of YT cells to $\beta$-1,3-glucan were comparable to previously reported receptor ligands such as LFA-1 and ICAM-1 ${ }^{38,39}$, and to H7-B6 (Fig. 3c), the canonical ligand for NKp30 expressed by K562 tumor cells, while there was only minimal binding to mannan or pustulan (Fig. 3a-c).

In contrast to $S$. cerevisiae, the distribution of glucans in the cell wall of Cryptococcus is reversed so that $\beta$-1,6-glucan exceeds $\beta$ 1,3 -glucan. While we observed strong binding of YT cells to $\beta$ 1,3 -glucan and to $S$. cerevisiae, we asked whether $\beta$-1,3-glucan of C. neoformans was required for NK cell binding. $C$. neoformans (strain B3501) were pretreated with caspofungin to reduce $\beta-1,3-$ glucan synthesis (Supplementary Fig. 2A-B). A single wild-type fungal cell (B3501) or $\beta$-1,3-glucan-depleted B3501 (B3501 $\Delta$ glucan) cell was glued to a cantilever and YT cells were adhered to the glass surface (schematic in Supplementary Fig. 4D-E, F). This

\footnotetext{
Fig. $1 \beta-1,3$-glucan is required for NKp30-mediated killing of Cryptococcus. a Flow cytometric analysis of cryptococcal cell wall/membrane (CCW/M) binding to $\mathrm{YT}$ cells as detected by mAb to $\beta-1,3-$ glucan vs. isotype control Ab. b Flow cytometric analysis of $\beta$-glucan binding to YT cell. YT cells were incubated with (left panel) or without (right panel) laminarin, a linear $\beta$-1,3-glucan with $\beta-1,6$-linkages. c Flow cytometric analysis of laminarihexaose binding to YT cells. YT cells were incubated with laminarihexaose (from curdlan, comprising only $\beta-1,3-g l u c a n$ ). $\mathbf{d}$ Comparison of binding of pustulan vs. $\beta$ 1,3-glucan to YT cells analyzed using flow cytometry. YT cells were incubated with pustulan (comprising only $\beta-1,6$-glucan) or $\beta-1,3-$ glucan (derived from $S$. cerevisiae). This experiment was performed twice. e Flow cytometric analysis of a recombinant NKp30-Fc fusion protein binding to $\beta$-glucan-conjugated beads ( $\beta-1,3-G B)$ compared to unconjugated polystyrene beads as control. NKp30-Fc on the beads was detected with anti-NKp30 antibody (1C01). f AntiNKp30 mAb (1C01) blocked NKp30 binding to $\beta-1,3-G B$. Recombinant NKp30 was incubated with 1 C01 before being applied to beads conjugated with $\beta$ 1,3-glucan. The presence of NKp30 on $\beta-1,3-G B$ was detected using the polyclonal anti-NKp30 antibody. $\mathbf{g}$ NKp30 binding to C. neoformans vs. C. albicans analyzed using flow cytometry. The experiment was performed twice. $\mathbf{h}$ Immunoprecipitation of NKp30 with $\beta-1,3-g$ lucan. YT cell lysate was incubated with $\beta$-1,3-glucan (laminarin) before being incubated with protein $\mathrm{G}$ beads that had been conjugated with a mAb against $\beta-1,3-g l u c a n$. i YT cell killing of $C$. neoformans (B3501) treated with caspofungin. Caspofungin concentrations were as indicated. \% reduction in CFU = CFU (B3501 with caspofungin alone) CFU (B3501 with corresponding caspofungin plus YT cells)/CFU (B3501 with caspofungin alone) $\times 100$ from raw data (Supplementary Fig. 3C). **, $p<$ 0.01. Data were analyzed using one-way ANOVA. All experiments were repeated three to five times on different days with similar results, unless specified otherwise. For flow cytometry analysis of 1,3-glucan, a mAb against $\beta$-1,3-glucan (Biosupplies, \#400-2, Australia) and FITC-labeled secondary Ab were used. $\beta-1,3-G B$ polystyrene beads conjugated with $\beta-1,3-$ glucan, unconjugated beads polystyrene beads without $\beta-1,3-$ glucan conjugation, rNKp30 recombinant NKp30 protein, anti-glucan mAb anti- $\beta-1,3-$ glucan, IP immunoprecipitate, cell lysate raw cell lysate, iso IgG isotype control
} 
a

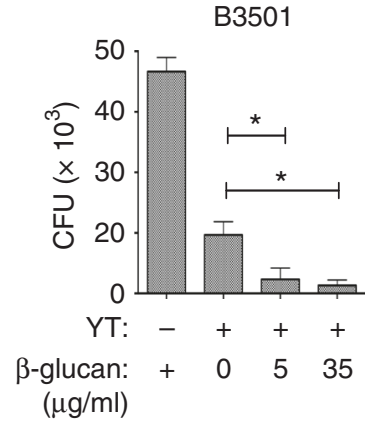

C

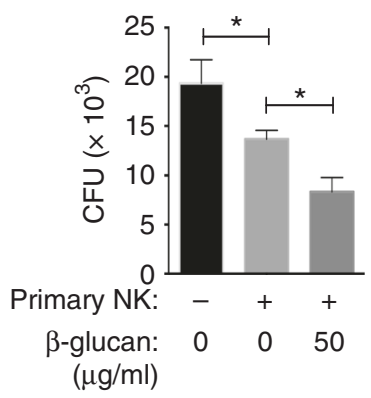

b

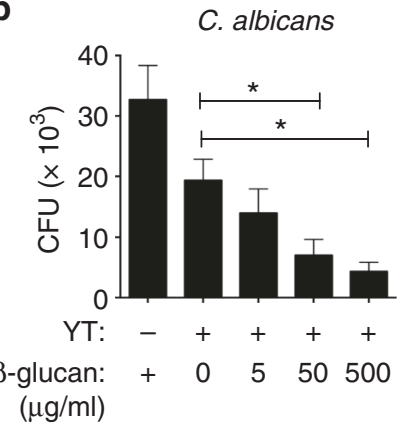

d

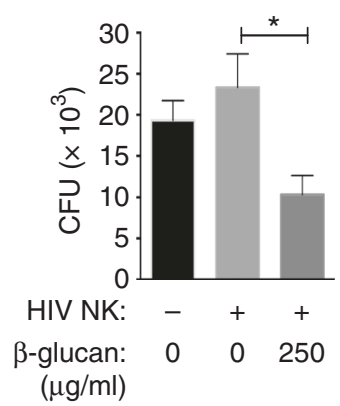

Fig. $2 \beta-1,3-$ glucan enhances killing by NK cells from healthy subjects and HIV patients. a YT cell killing of $C$. neoformans in the presence of $\beta-1,3-$ glucan (CFU). YT cells were treated with 0,5 , and $35 \mu \mathrm{g} / \mathrm{ml}$ of soluble $\beta$ 1,3-glucan (laminarin derived from L. digitata) for $4 \mathrm{~h}$ before the addition of C. neoformans (strain B3501). CFU were determined in quadruplicate \pm SEM. $\mathbf{b}$ YT cell killing of $C$. albicans in the presence of increasing doses of $\beta$ 1,3-glucan $(0,5,50$, and $500 \mu \mathrm{g} / \mathrm{ml})$. c Primary NK cell killing of $C$. neoformans (B3501) in the presence of $\beta-1,3$-glucan $(0$ and $50 \mu \mathrm{g} / \mathrm{ml})$ for NK cells from healthy subjects. $\mathbf{d}$ Killing of $C$. neoformans (B3501) by primary NK cells from HIV-infected individuals in the presence of $\beta-1,3-$ glucan $(0$ and $250 \mu \mathrm{g} / \mathrm{ml})$. All experiments were performed for three to five times with similar results. ${ }^{\star}, p<0.05$. Data were analyzed using oneway ANOVA

resulted in a marked reduction in the binding force to B3501 ${ }^{\Delta \text { glucan }}$ (Fig. 3d, middle and right panels) compared to wild-type B3501 (Fig. 3d, left. $p<0.01$, by $T$-test), revealing that $\beta$-1,3-glucan is required for binding of $C$. neoformans to YT cells. To further confirm that $\beta$-1,3-glucan binds to YT cells via NKp30, siRNA was used to knock down NKp30 in YT cells ${ }^{15}$, and binding of $\beta$-1,3-glucan-coated beads ( $\beta-1,3-\mathrm{GB})$ to these YT cells was measured by SCFS/atomic force microscopy(AFM). Accumulated binding forces showed that NKp30 knockdown abrogated binding of $\beta-1,3-\mathrm{GB}$ to YT cells (Fig. 3e). It is known that other receptors that may recognize $\beta$-1,3-glucan, although only dectin-1 has been identified as the primary receptor on leukocytes (reviewed in ref. $^{40}$ ). Our data showed that dectin-1 is not expressed by YT cells or primary NK cells (Supplementary Fig. 5A). Taken together, we conclude that the binding of $\beta-1,3-$ glucan to YT cells is through NKp30.

$\beta$-1,3-glucan binds to NKp30 as demonstrated by AFM. Having demonstrated that $\beta$-1,3-glucan binds to YT cells and that $\beta-1,3-$ glucan is required for binding of $C$. neoformans to NK cells, we sought to determine whether NKp30 was sufficient for binding using AFM. To do so, $\beta-1,3-\mathrm{GB}$ were fixed to a cantilever tip and a glass surface was coated with the recombinant ectodomain of NKp30 (schematic in Supplementary Fig. 4G). Representative F-D curves (Fig. 3f), accumulated binding forces (Fig. 3g, left panel), and mean forces (Fig. 3g, right panel) showed that NKp30 bound with much greater force to $\beta-1,3-\mathrm{GB}$ than to controls $(p<$ 0.01 , by one-way ANOVA). These data reveal that NKp30 was sufficient to explain the binding force, and is a major binding partner for $\beta$-1,3-glucan.

In some experiments, a recombinant $\mathrm{NKp} 30-\mathrm{Fc}$ fusion protein was used. We considered the possibility that $\beta$-1,3-glucan bound to the Fc portion of this fusion protein. To test for this possibility, we compared binding to another recombinant $\mathrm{Fc}$ fusion protein, CD28-Fc. AFM showed that binding forces of CD28-Fc were comparable to other controls, while forces for NKp30-Fc was significantly higher (Fig. $3 \mathrm{~h}, p<0.01$, by $T$-test) indicating $\beta$ - 1,3 glucan binding to $\mathrm{NKp} 30-\mathrm{Fc}$ is through $\mathrm{NKp} 30$ rather than $\mathrm{Fc}$.

$\boldsymbol{\beta}-1,3$-glucan induces granule polarization in NK cells. NK cell killing of Cryptococcus is a perforin-mediated process ${ }^{15}$, in which granules traffic to the immunological synapse (IS) to release their cytolytic cargo and kill the target cells. To determine whether $\beta$-1,3-glucan was sufficient to elicit NK cell granule trafficking in real-time, YT cells transfected with microtubule-associated protein 4-GFP (YT-MAP4-GFP cells) were labeled with Lysotracker Red DND-99 to visualize granule trafficking, and $\beta$-1,3-GB were used as the stimulus. Time-lapse video of YT cells in contact with a bead was obtained to capture granule movement. Granules in the YT cell converged toward one another (Fig. 4a, upper two rows, Fig. $4 \mathrm{~b}$ and Supplementary movie 1 ) and polarized toward the $\beta-1,3-G B$ (Fig. $4 \mathrm{a}$, upper two rows, Fig. $4 \mathrm{c}$ and Supplementary movie 1), but not to control beads (Fig. 4a, third row, and Fig. 4b, c and Supplementary movies 2-3). To confirm that NKp30 was required for YT cell granule polarization toward $\beta$-1,3-glucan, siRNA was used to knock down NKp30 in YT cells, and live cell imaging was performed. YT cells with reduced expression of $\mathrm{NKp} 30$ failed to converge and polarize granules in response to $\beta$ 1,3-GB (Supplementary movie 7) compared with wild-type YT cells, indicating that NKp30 is critical for the YT cell response to $\beta$-1,3-glucan.

$\beta$-1,3-glucan stimulates perforin polarization and release. When NK cells form a synapse with Cryptococcus, perforin is polarized to the IS ${ }^{15}$. To determine whether $\beta$-1,3-glucan was sufficient to stimulate perforin polarization, YT cells were incubated with $\beta-1,3-\mathrm{GB}$, and labeled with anti-perforin antibody. Representative photomicrographs (Fig. 4d) and measurements of the distance between perforin and the IS (Fig. 4e) showed that perforin was polarized at the IS in more than $80 \%$ of the YT cells in contact with $\beta-1,3-\mathrm{GB}$, but not to control beads (Fig. $4 \mathrm{f}$ ). These data support the conclusion that NK cells polarize perforin to the IS in response to $\beta$-1,3-glucan as it does during fungal killing.

We previously showed that perforin expression is markedly increased after stimulation with $C$. neoformans ${ }^{15,41}$. To determine whether $\beta$-1,3-glucan was sufficient to increase perforin expression, YT cells were incubated with soluble $\beta$-1,3-glucan. We found that both soluble $\beta$-1,3-glucan and immobilized $\beta-1,3-\mathrm{GB}$ enhanced perforin levels (Fig. 4g). Soluble and immobilized $\beta-1,3-$ glucan also resulted in increased perforin levels in the culture media (Fig. 4h), suggesting increased degranulation and release.

$\beta-1,3$-glucan stimulates NKp30 clustering at the IS. In addition to increased perforin expression, there are a number of possible mechanisms by which $\beta$-1,3-glucan could enhance NK cell effector function including increased receptor expression, synapse formation, and the magnitude of signaling events. We examined NKp30 expression after stimulation by $\beta$-1,3-glucan. Flow cytometry showed that $\beta$-1,3-glucan produced a time-dependent increase in NKp30 expression on YT cells (Fig. 5a, b, and 
Supplementary Fig. 5B-C). This provides a possible mechanism, whereby greater signaling occurs because of enhanced receptor expression.

We previously showed that Cryptococcus forms a synapse with NK cells, which includes clustering of $\mathrm{NKp} 30$ at the area of contact prior to perforin polarization ${ }^{15}$. To determine whether NKp30 clustered in response to $\beta$-1,3-glucan, YT cells were mixed with $\beta-1,3-\mathrm{GB}$ and labeled with anti-NKp30 Ab. Photomicrographs showed that NKp30 was clustered at the synapse between YT and $\beta-1,3-\mathrm{GB}$ (Fig. $5 \mathrm{c}$, d). More than $80 \%$ of the YT cells in contact with $\beta-1,3-\mathrm{GB}$ had NKp30 clustering (Fig. 5e), revealing that $\beta$-1,3-glucan not only induced NKp30 expression, but also induced its relocation to the IS, which would provide a signaling platform that enhanced NK cell killing.
To determine the proximity of clustered NKp30 to the IS, we used total internal reflection fluorescence (TIRF) microscopy to visualize events at a pre-defined distance $(80-100 \mathrm{~nm})$ from the interface between NK cells and $\beta$-1,3-glucan. In conjunction with TIRF, spinning disc (SD) confocal microscopy was used to capture images from the top to the bottom of the cells. YT cells were loaded into a glass chamber that had been coated with $\beta-1,3$ glucan or mannan (Supplementary Fig. 4A). YT cells that came into contact with $\beta$-1,3-glucan or control surfaces showed 1C01 labeling throughout the cells using SD; however, only the cells in contact with the $\beta$-1,3-glucan-coated surface showed increased 1C01 labeling at the interface by TIRF microscopy (Fig. 5f). Quantitative image analysis of the pixel intensities from NKp30 labeling was performed as illustrated (Supplementary Fig. 6A-B).

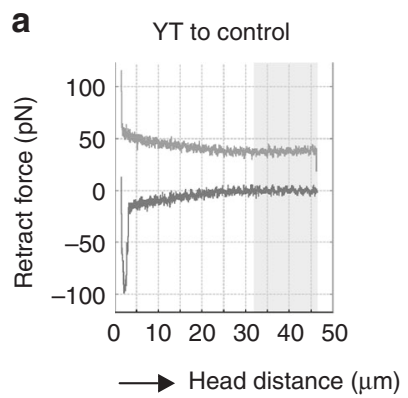

b

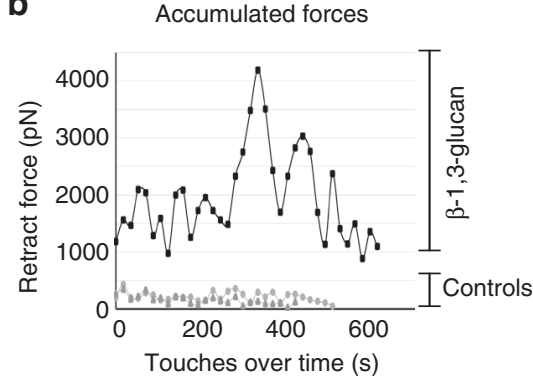

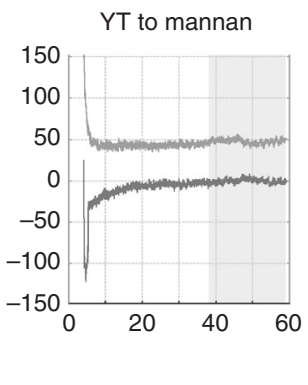

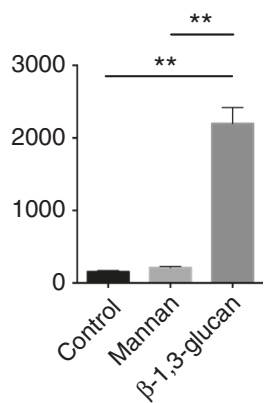

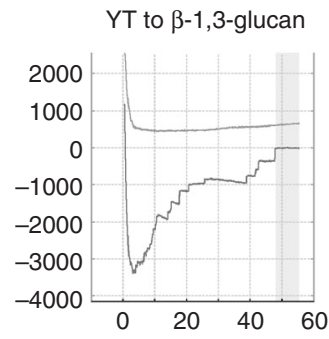

C

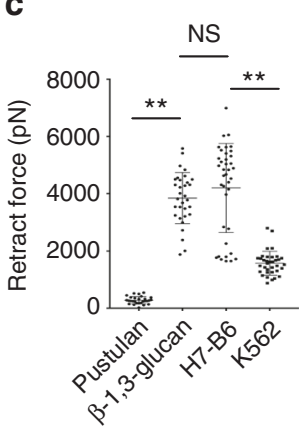

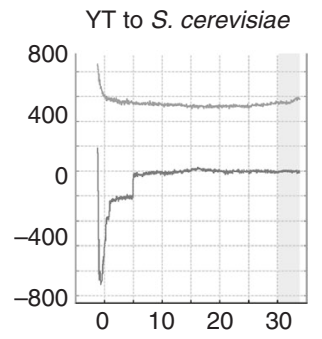

e
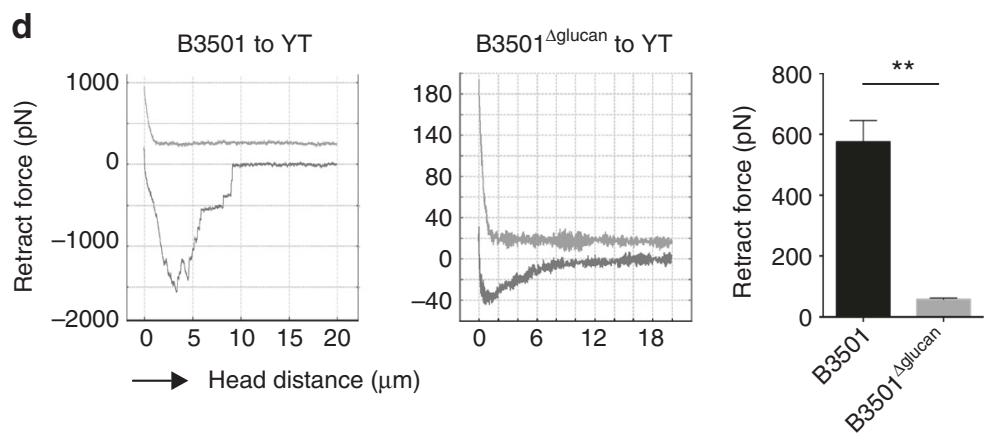

f
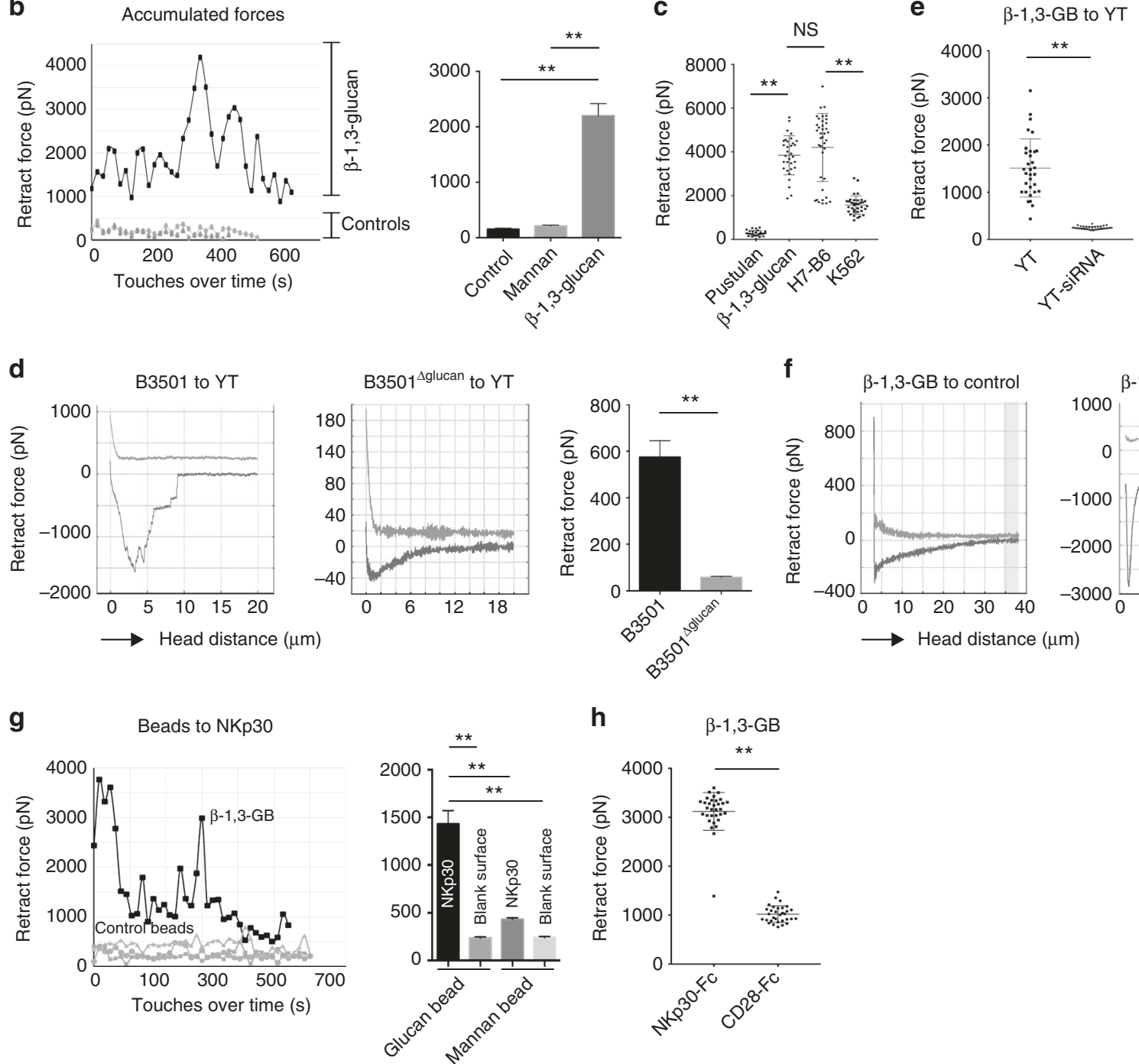
The intensity of NKp30 labeling on TIRF images were up to five times higher in YT cells adhered to $\beta$-1,3-glucan than to the control surface (Fig. 5g), suggesting that NKp30 was clustering at the cell surface in response to $\beta$-1,3-glucan leading to enhanced NK cell killing.

$\beta-1,3$-glucan activates Src family kinase in NK cells. Since the Src family kinases (SFK) Fyn and Lyn are required for perforin polarization and NK cell cryptococcal killing ${ }^{42}$, we asked whether $\beta$-1,3-glucan stimulated SFK activation in NK cells. YT cells were incubated with $\beta-1,3-\mathrm{GB}$, mannan-conjugated, or unconjugated beads as controls. Using an antibody that detects Tyr416 phosphorylation of SFK, western blot showed activation in YT cells in response to $\beta-1,3-\mathrm{GB}$, but not to controls (Fig. 6a).

To address whether NKp30 ligation and downstream SFK activation were required for NKp30 clustering, we used $1 \mathrm{C} 01$ to block NKp30 binding or used dasatinib to inhibit Src family (Tyr416) activation ${ }^{43}$. Representative photomicrographs of immunocytochemistry (Fig. 6b) and quantitative assessment of the proximity of NKp30 to the synaptic radius (Fig. 6c) showed that NKp30 clustering was abrogated by either $1 \mathrm{C} 01$ or dasatinib compared to isotype-matched IgG or DMSO controls (Fig. 6c,d), indicating that NKp30 clustering required NKp30 and SFK signaling.

Live cell imaging was used to determine whether SFK signaling was required for granule polarization in response to $\beta$-1,3-glucan. Lysotracker-labeled YT-MAP4-GFP cells were pretreated with $1 \mathrm{C} 01$ to block NKp30, or with dasatinib to interrupt SFK signaling. Video images showed that granule polarization at the IS was abolished by treatment with either 1C01 (Fig. 6e, upper panel, Fig. 6f, left panels, Supplementary movie 4) or dasatinib (Fig. 6e, middle panel, Supplementary movie 5) compared to control (Fig. 6f, lower panel and Supplementary movie 6, Fig. 6f, right panel). Thus, NKp30 and SFK signaling was required for NKp30 receptor clustering and granule trafficking and polarization at the IS in response to $\beta$-1,3-glucan.

\section{Discussion}

We have made four major observations: 1) $\beta$-1,3-glucan bound to recombinant NKp30 and to NKp30 expressed on YT cells; 2) Blocking $\beta$-1,3-glucan synthesis in $C$. neoformans inhibited NK cell binding and killing of Cryptococcus; 3) $\beta$-1,3-glucan activated $\mathrm{NK}$ cells to increase expression of $\mathrm{NKp} 30$ and perforin, induced NKp30 clustering, granule polarization at the IS, as well as perforin release and enhanced killing of Cryptococcus and C. albicans; and 4) $\beta$-1,3-glucan was able to restore defective NK cell cryptococcal killing from HIV-infected individuals.
We had previously identified NKp30 as an Ig-like transmembrane PRR for fungi ${ }^{15}$, and now demonstrate that $\beta$-1,3-glucan is the fungal PAMP for NKp30. $\beta$-glucans have been observed to protect against bacteria, virus, Candida, and parasite in experimental models ${ }^{44-49}$, and appears to be beneficial for high-risk surgical patients ${ }^{50-52}$. However, most prior studies were focused on innate immunes cells other than NK cells and it is not clear to what extent activation of NK cells contributed to enhanced host defense in the prior studies.

Our results demonstrate a direct mechanism, whereby complex polymers such as laminarin or $\beta$-1,3-glucan from Euglena crosslink and activate NKp30, although we have not excluded the possibility that other receptors might bind to $\beta$-glucan and play a role. Dectin-1, a C-type lectin receptor, is unlikely to play a role since it is found on macrophages, monocytes, dendritic cells, neutrophils, eosinophils, B cells, and a subpopulation of T cells, but not on NK cells ${ }^{53,54}$ or YT cells (Supplementary Fig. 5A). Complement receptor 3 is an integrin $\left(\mathrm{CD} 11 \mathrm{~b} / \mathrm{CD} 18, \alpha_{M} \beta_{2^{-}}\right.$ integrin) expressed by NK cells and used to recognize $\beta$-glucan and mediate cytotoxicity against tumor cells ${ }^{55}$. However, our previous studies failed to identify a role for CR3 in NK cell killing of Cryptococcus ${ }^{56}$.

These studies have possible implications for translation to patients. We previously showed that IL-12 restored cryptococcal killing by NK cells from HIV-infected patients ${ }^{36}$. However, IL-12 is costly and associated with side effects that limit its therapeutic use. Since $\beta-1,3$-glucan is generally not in the blood of patients with cryptococcosis ${ }^{57,58}$, we might anticipate that NK cells would not have been pre-activated by $\beta-1,3$-glucan, and that NK cells would be responsive to therapy. If $\beta-1,3$-glucan could activate NK cell in vivo, it might enhance host defense to fungal infection, including HIV-infected patients who are at risk of cryptococcosis. In addition, although caspofungin is not active against C. neoformans at clinically relevant concentrations ${ }^{59}$, and would not be used for treatment of cryptococcosis, treatment of fungi with caspofungin compromised the effectiveness of NK-mediated killing of Cryptococcus, suggesting that inadvertent treatment of cryptococcosis with an echinocandin would not only lack antimicrobial activity, but would also impair host defense to this pathogen.

In summary, we identified $\beta$-1,3-glucan as the PAMP for the cytotoxic PRR, NKp30, and identified the mechanism by which $\beta$-1,3-glucan triggered and enhanced NK cell killing of fungi. Furthermore, our data showed that $\beta$-1,3-glucan can activate NK cells and restore the defective response in NK cells from HIVinfected patient, which may present therapeutic opportunities.

Fig. 3 NKp30 interaction with $\beta$-1,3-glucan revealed by AFM. a Representative force-distance (F-D) curves of YT cell binding to $\beta$-1,3-glucan. A single $Y T$ cell was attached to the cantilever tip. Mannan, media, $\beta-1,3-$ glucan, or $S$. cerevisiae were coated on poly-L-lysine-treated glass. Peak downward deflection is the force of disruption. $\mathbf{b}$ Binding forces of $Y T$ cells to $\beta-1,3-g$ lucan vs. controls. A single YT cell was brought into contact and was detached from the matrix 20-30 times. At least three spots on the glass were tested per YT cell. Left panel: maximum binding forces from each valid contact over time. Right panel: average binding forces of $\mathrm{YT}$ cells to $\beta$-1,3-glucan, mannan, and blank glass control. c Binding forces of $\mathrm{YT}$ cell to $\beta$-1,3-glucan vs. H7-B6, and pustulan. A single $Y T$ cell was brought into contact and was detached from the matrix multiple times. Three to six areas on the coated glass were tested per $Y T$ cell. $\mathbf{d}$ F-D curves of C. neoformans binding to YT cells. A single B3501 cell was fixed to a cantilever tip, and YT cells were adhered to either uncoated or poly-Llysine glass. Left panel: binding of untreated C. neoformans strain B350, middle panel binding of caspofungin-treated, $\beta$-1,3-glucan-deficient $C$. neoformans

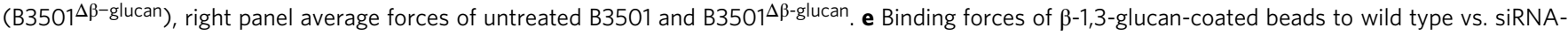
NKp30-treated YT cells. $\beta$-1,3-glucan-coated beads ( $\beta-1,3-G B)$ were glued to a cantilever, and the cells were adhered to a poly-L-lysine-coated ibidi dish. $\mathbf{f}$ Representative F-D curves of binding forces of polystyrene beads conjugated with or without $\beta$-1,3-glucan to recombinant NKp30 (right panel) vs. control glass surface (left panel). $\mathbf{g}$ Accumulated binding forces of beads conjugated with or without $\beta$-1,3-glucan to NKp30 or controls (left panel); average binding forces of beads conjugated with or without $\beta-1,3-g l u c a n$ to NKp30 or controls (right panel), note change of scale on vertical axis. $\mathbf{h}$ Binding forces of $\beta$-1,3-glucan-coated beads ( $\beta-1,3-G B)$ to recombinant Fc chimera NKp30 vs. recombinant Fc chimera CD28. All experiments were repeated two to four times on different days with similar results. Glucan $\beta$-1,3-glucan, glucan beads $\beta$-1,3-glucan-conjugated polystyrene beads, isotype control IgG, NKp30 polyclonal anti-NKp30 antibody. ${ }^{\star \star}, p<0.01$. Data were analyzed using one-way ANOVA b, c, g or T-test d, e, h. pN, piconewton 

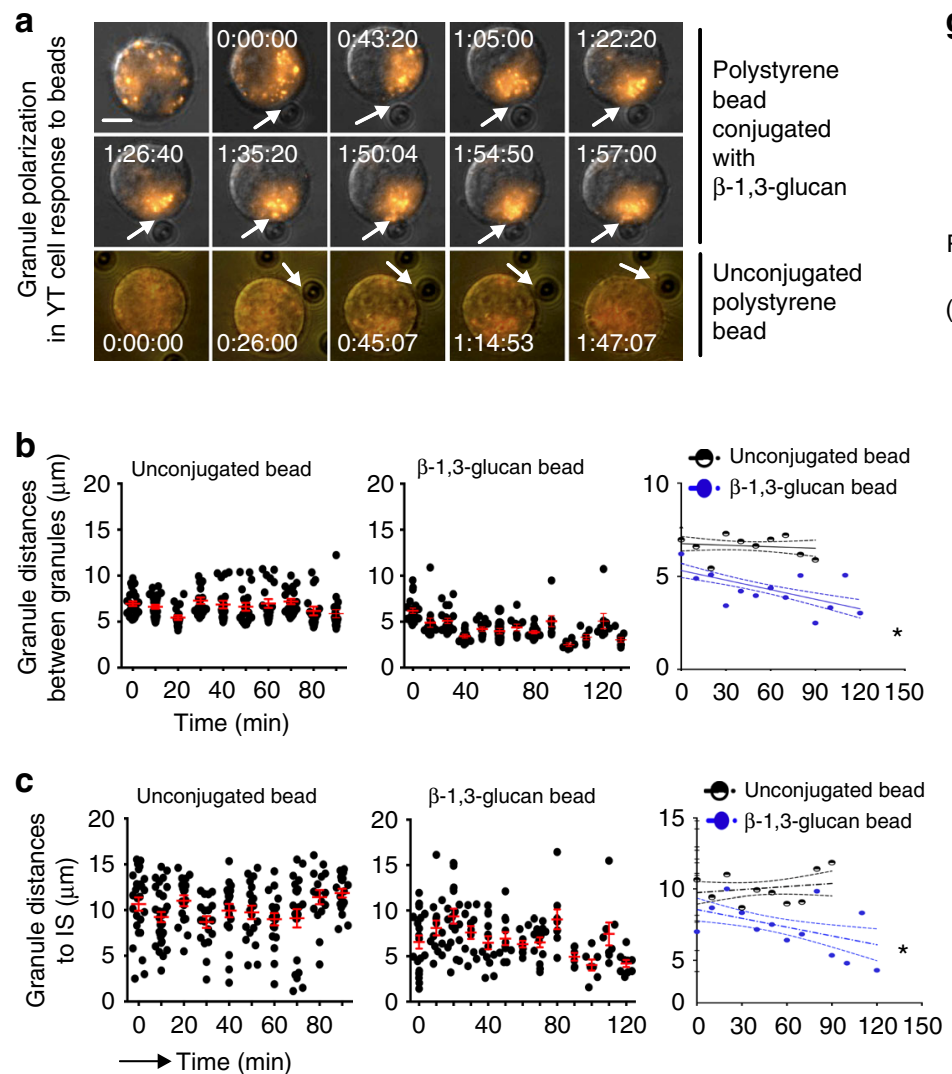

d

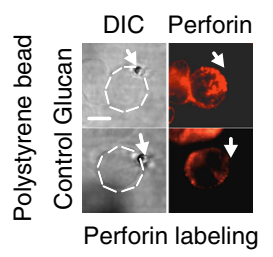

e

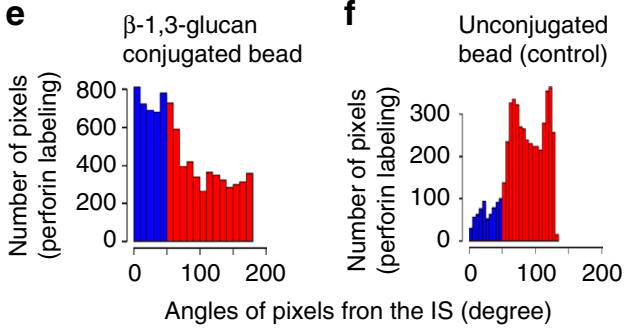

g

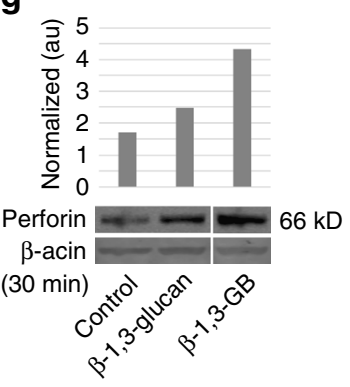

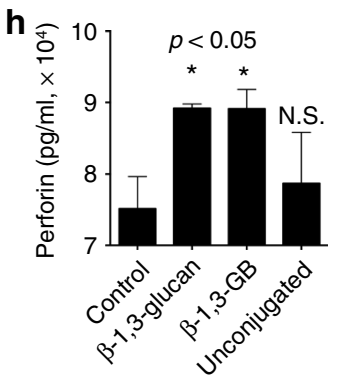

Fig. $4 \beta$-1,3-glucan stimulates granule polarization in NK cells. a Representative images from real-time live cell imaging (Supplementary movie 1) of granule polarization toward the IS in NK cells in response to $\beta-1,3-G B$. YT cells were loaded with Lysotracker Red to visualize granules and mixed with $\beta-1,3-$ glucanconjugated, or control beads. The experiment was repeated nine times with eight having similar results. Bar $=5 \mu$ m. $\mathbf{b}$ Distance between granules (black circles) and mean distance between granules (red symbols) from panel a. The mean distance between granules with $95 \%$ confidence intervals is shown in the right panel. c Distances between granules and the IS, defined as the center of the point of contact between a YT cell responding to unconjugated beads (left panel) or $\beta-1,3$-glucan beads (middle panel) from panel a. The mean distance from granules to IS with $95 \%$ confidence intervals is shown in the right panel. Distances were determined at times throughout the video and do not necessarily corresponding to times in $\mathbf{a}$. $\mathbf{d}$ Perforin expression and polarization at the IS between YT cells and $\beta-1,3-G B$ in a representative single YT cell (20 cells analyzed). Experiments were repeated at least three times with similar results. Bar $=5 \mu \mathrm{m}$. e Proximity of perforin labeling to the radius defined by the center of the IS with the $\beta-1,3-G B$. Polarization was defined when the peak of fluorescence was at an angle $<45^{\circ}$ (blue bars in the left). f Proximity of perforin labeling to the synaptic radius with unconjugated bead. Polarization was defined when the peak of fluorescence was at an angle $<45^{\circ}$ (blue bars in the left). $\mathbf{g}$ Western blot analysis of perforin expression in $\mathrm{YT}$ cells in response to soluble and $\beta-1,3-G B$. The experiment was repeated at least three times with similar results. $\mathbf{h}$ Perforin levels released in the culture media assessed using ELISA. YT cells were treated with soluble $\beta-1,3-$ glucan, $\beta-1,3-G B$, or control. Quadruplicate determinations were performed in two experiments with similar results. N.S. non-significant. ${ }^{*}, p<0.05$. Data were analyzed using one-way ANOVA $(\mathbf{b}, \mathbf{c}, \mathbf{h})$

\section{Methods}

Cells and microorganisms. YT cells ${ }^{60}$ (NK cell line, a gift from C. Clayberger, Stanford University, Stanford, CA), YT-MAP4-GFP cells were derived from

YT cells transfected with microtubule-associated protein 4 (MAP4)-GFP (unpublished data). Primary NK cells were isolated from the blood of healthy and HIVinfected individuals using EasySep (StemCell, \#19055) as described ${ }^{15}$. Primary NK cells were from HIV-infected patients receiving antiretroviral therapy with CD4 +counts between 300 and 700 cells $/ \mu$ and no detectable viral load $(<40$ copies $/ \mathrm{ml})$ from the Southern Alberta Clinic. K562 cells (ATCC CCL-243) are hematopoietic malignant cells (a gift from Dr. Oliver Bathe, University of Calgary). These cells were maintained in complete RPMI 1640 medium supplemented with $10 \%$ FCS, $1 \%$ pen-strep, $1 \%$ sodium pyruvate (Invitrogen, GIBCO 11360 ), and $1 \%$ nonessential amino acids (all from Invitrogen) at $37^{\circ} \mathrm{C}$ and $5 \% \mathrm{CO}_{2}$. The recombinant Fc chimera H7B6 was purchased from R\&D Systems (8984-B7). Recombinant Fc chimera CD28 was purchased from BioLegend (NM_006139). C. neoformans strain B3501 (ATCC 34873) and C. albicans (ATCC 58716, serotype A, formerly LUMC 101) were cultured at $32{ }^{\circ} \mathrm{C}$ overnight in Sabouraud dextrose broth (BD, Cat. 238230) with shaking until they were at $\log$ phase of growth before the experiment. C. neoformans strain B3501 was treated with caspofungin (SML0425, SigmaAldrich) at $37^{\circ} \mathrm{C}$ for $5-6 \mathrm{~h}$ to block $\beta-1,3$-glucan synthesis (named B3501 $1^{\Delta \text { glucan }}$ ). 
a

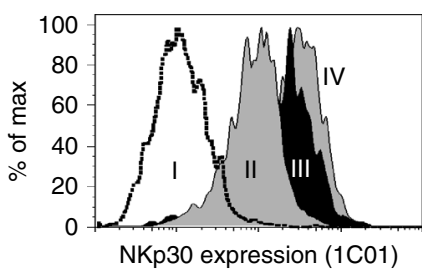

I: Isotype IgG (MFI: 17.5) III: YT + laminarin (MFI: 264)

II: YT alone (MFI: 110) IV: YT + Euglena (MFI: 365) b

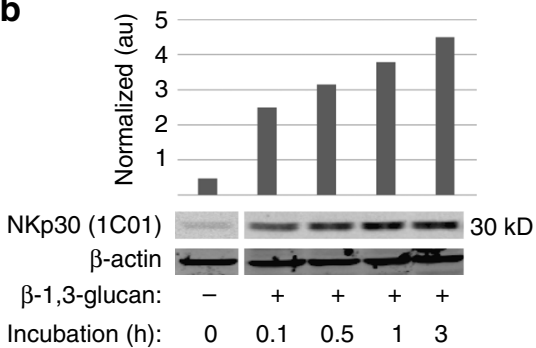

C

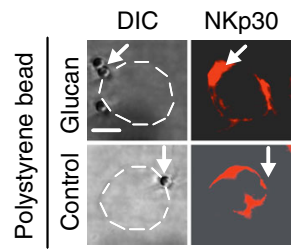

e

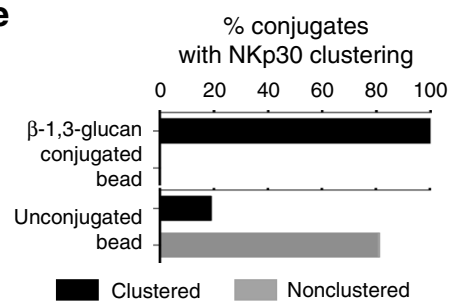

g

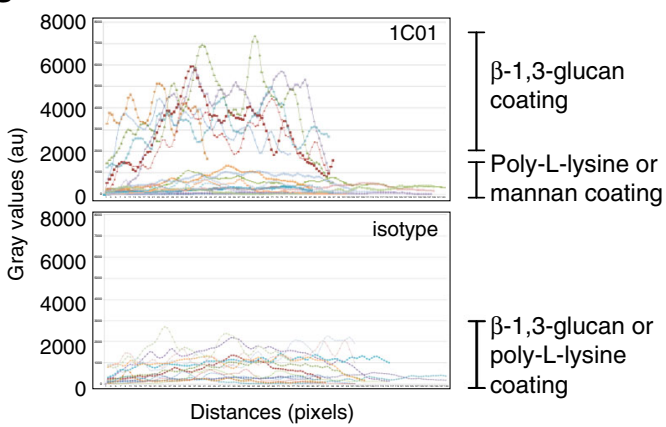

d

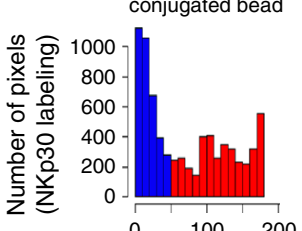

Angles of pixels from the IS (degree)

\section{f}

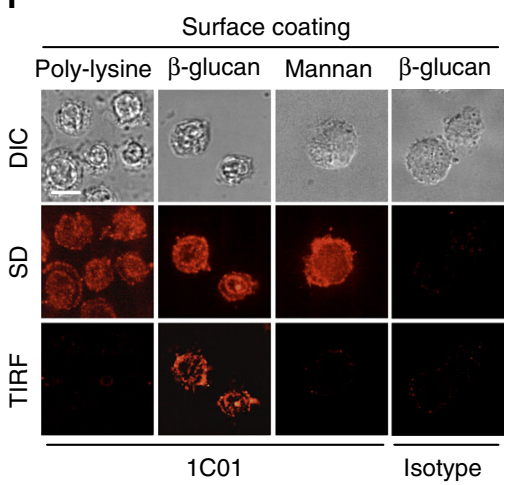

Fig. $5 \beta-1,3-$ glucan increases expression and clustering of NKp30 in NK cells. a NKp30 expression in YT cells in response to $\beta-1,3-g l u c a n . ~ Y T$ cells were treated with $\beta-1,3$-glucan (III and IV) and expression of NKp30 using 1C01 was compared to untreated cells (I), or untreated cells labeled with isotype control (II). b Western blot analysis of NKp30 expression in YT cells in response to $\beta-1,3$-glucan. YT cells were incubated with or without $\beta$-1,3-glucan for various times. The experiment was repeated three times with similar results. c NKp30 labeling at the synapse between a representative single $\mathrm{YT}$ cell and a single $\beta-1,3$-glucan-conjugated bead. 1C01 was used to label NKp30. The location of the bead is shown with a white arrow. Bar $=5 \mu \mathrm{m}$. $\mathbf{d}$ Proximity of NKp30 expression in relation to the radius defined by the center of the IS ( $n=21$ cells) in panel $\mathbf{c}$. The method for determining the angle is illustrated in Supplementary Fig. 6C. Clustering was defined when the peak of fluorescence was at an angle $<45^{\circ}$ (blue bars in the left). e Frequency of NKp30 clustering of all YT cell conjugates with $\beta$-1,3-glucan-conjugated or unconjugated beads as analyzed in panel $\mathbf{c}$, as defined in panel $\mathbf{d}$. $\mathbf{f}$ TIRF microscopic analysis of NKp30 expression at the interface between YT cells and $\beta$-1,3-glucan or control. YT cells were loaded into a glass chamber that had been coated with $\beta$-1,3glucan or mannan incubated at $37^{\circ} \mathrm{C}$ for $30 \mathrm{~min}$, fixed and stained for NKp30 using $1 \mathrm{CO}$. Bar $=10 \mu \mathrm{m}$. $\mathbf{g}$ Fluorescent intensity of NKp30 from panel $\mathbf{f}$ in arbitrary units. Distance is defined in Supplementary Fig. 6A-B. All experiments were repeated three times with similar results. TIRF total internal reflection fluorescence, poly-L-lysine coating alone or mannan coating served as control, Au arbitrary unit, Arrow bead, SD spinning disc microscopy, DIC differential interference contrast digital image, IS immunological synapse

The reduced levels of $\beta$-1,3-glucan in B3501 ${ }^{\Delta \text { glucan }}$ were confirmed using anti- $\beta$ 1,3-glucan antibody (Biosupplies \#400-2, Australia) and flow cytometric analysis. Polystyrene beads conjugated with $\beta-1,3$-glucan were prepared as described ${ }^{33}$. Sources for $\beta$-1,3-glucan: S. cerevisiae (Sigma G5011); barley (Sigma, G6513); Euglena gracilis (Sigma, 89862); laminarin (Sigma, L9634); laminarihexaose from curdlan (Megazyme, O-LAM6); and pustulan (Carbomer, 4-00507). Monoclonal antibodies were $1 \mathrm{C} 01$ to NKp30 ${ }^{15}$, anti-NKp30 antibody (R\&D, clone\# 210845), anti-perforin antibody (eBioscience, Clone eBioBOR21), and anti- $\beta$-1,3-glucan
(Biosupplies \#400-2, Australia). Rabbit anti-phospho-Src family kinase (Cell Signaling Y416); mouse anti-Fyn (BD Transduction Laboratories).

Detection of $\boldsymbol{\beta}$-1,3-glucan and NKp30 by flow cytometry. For NK cell surface detection of $\beta-1,3$-glucan, YT cells were incubated with a preparation of cryptococcal cell wall/membrane (CCW/M) or purified $\beta$-1,3-glucan (e.g., laminarin, laminarihexaose, curdlan) or controls (pustulan) at $4{ }^{\circ} \mathrm{C}$ overnight and detected by 

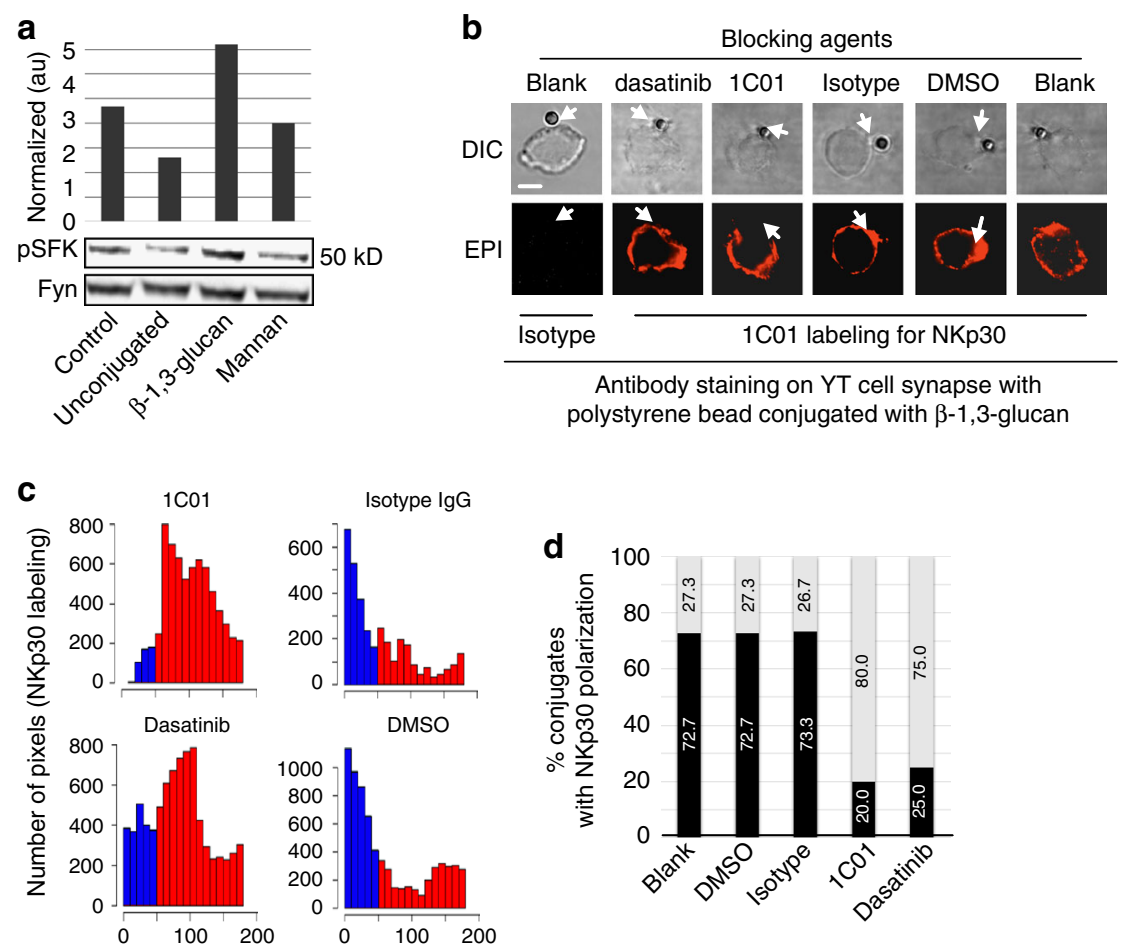

Angles of pixels from the IS (degree)

e
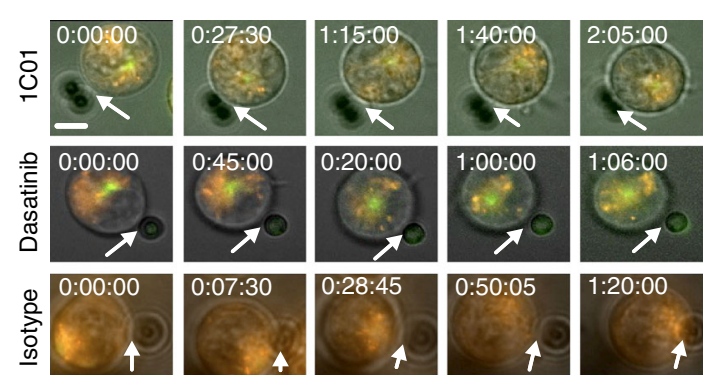

$\mathbf{f}$
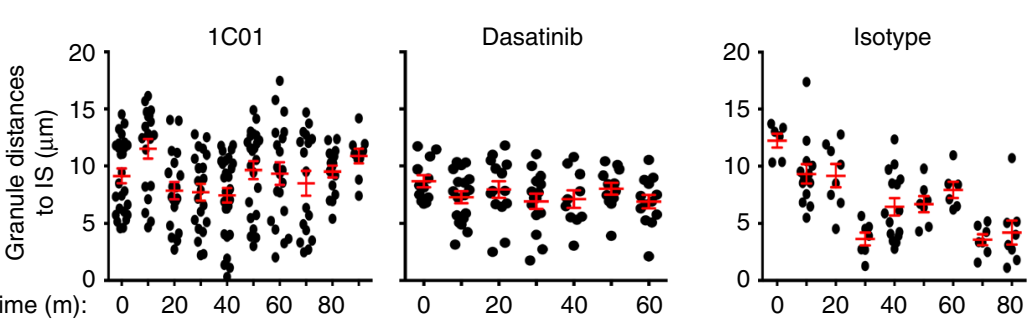

Fig. $6 \beta$-1,3-glucan induces SFK signaling in NK cell response. a Western blot analysis of SFK phosphorylation in YT cells in response to $\beta$-1,3-GB vs. controls. The experiment was done twice with similar results. pSFK phosphorylated SFK (Src Family Kinase, Y416), control YT cells alone. b NKp30 expression in YT cells in response to $\beta-1,3-\mathrm{GB}$ in the presence of $12.5 \mu \mathrm{g} / \mathrm{ml}$ of $1 \mathrm{C} 01,50 \mathrm{nM}$ dasatinib, or controls (isotype IgG or DMSO). Cells and beads were labeled with 1C01. Arrow indicates interface between the YT cell and beads. The experiment was repeated at least three times on different days with similar results. Bar $=5 \mu \mathrm{m}$. c Proximity of NKp30 expression in relation to the radius defined by the IS (representative of $n=15$ cells analyzed for 1 C01 or 20 cells for dasatinib) in panel $\mathbf{b}$. Clustering was defined when the peak of fluorescence was at an angle less than $45^{\circ}$ (blue bars in the left). $\mathbf{d}$ Frequency of NKp30 clustering in YT cell that had formed conjugates with beads in panel $\mathbf{b}$ as defined in panel $\mathbf{c}$. e Representative photomicrographs derived from timelapse videos of YT cells in response to polystyrene beads conjugated with $\beta-1,3$-glucan in the presence or absence of 1 C01 or dasatnib (Supplementary movies 4-7), respectively. YT cells were loaded with Lysotracker and incubated with 1C01, dasatinib, or control medium before being incubated with $\beta-1,3-$ GB. The experiment was performed for three times with similar results. Bar $=5 \mu \mathrm{m}$. f Distances from each granule to the IS derived from data as in panel a (red symbols indicate mean \pm SEM)

$\mathrm{mAb}$ to $\beta$-1,3-glucan (Biosupplies, \#400-2, Australia). Flow cytometric analysis was performed using Guava easyCyte Flow Cytometer (Guava inCyte v5.2, Millipore Sigma). For flow cytometric analysis of NKp30 binding to $\beta$-1,3-glucan, $\beta$-glucanconjugated beads were incubated with a recombinant NKp30-Fc fusion protein $(\mathrm{R}$ \& D Systems, \# O95944) at $4{ }^{\circ} \mathrm{C}$ overnight, NKp30-Fc on the beads was detected with anti-NKp30 antibody (1C01). For NKp30 binding to C. neoformans and C. albicans, the fungi were incubated with a recombinant ectodomain NKp $30^{61}$ at $4^{\circ}$ $\mathrm{C}$ overnight, and detection of NKp30 was performed using a polyclonal anti-Kp30 antibody (Abnova, PAB17794). 
Immunoprecipitation assay and western blot. Protein G beads (Dynabeads 100.07D, Thermo Fisher) were used to immunoprecipitate NKp30. Briefly, YT cells were lysed and incubated with $\beta$-1,3-glucan (laminarin) at $4{ }^{\circ} \mathrm{C}$ for $1-3$ days. The protein $\mathrm{G}$ beads were conjugated with antibody against $\beta$-1,3-glucan or isotype antibody at room temperature for $10 \mathrm{~min}$. These beads were incubated with the mixture of YT cell lysate with $\beta$-1,3-glucan at room temperature for $20 \mathrm{~min}$. The immunoprecipitates (complex of NKp30 with its ligand) were eluted from the beads and resolved in NuPAGE Novex 4-12\% Bis-Tris Gel (Thermo Fisher, NP0321BOX)

For SFK signaling studies, YT cells were treated with beads conjugated with or without $\beta$-1,3-glucan at $37^{\circ} \mathrm{C}$ for $5 \mathrm{~min}$, or for the indicated times with soluble $\beta$ 1,3-glucan. Cell lysates were collected and resolved in NuPAGE Novex 4-12\% BisTris Gel (Thermo Fisher). Proteins in the gel were transferred to a nitrocellulose membrane and blotted for NKp30 with 1C01 or anti-phosphorylated SFK (pSFK, Y416).

Antifungal activity assay. Unless otherwise specified, $2 \times 10^{5} \mathrm{YT}$ cells or primary NK cells were co-cultured with C. neoformans or C. albicans at a starting effector to target ratio of 100-200:1 in $200 \mu \mathrm{l}$ complete RPMI 1640 medium per well in 96-well plates (Costar) at $37^{\circ} \mathrm{C}$. Colony forming units (CFU) were determined as previously described ${ }^{15}$. To investigate the effects of $1 \mathrm{C} 01$ or dasatinib (Sigma-Aldrich, CDS023389) on the YT cell response to $\beta-1,3-\mathrm{GB}$, mannan, or unconjugated beads, YT cells were pretreated with $1 \mathrm{C} 01$ or dasatinib at $37^{\circ} \mathrm{C}$ for $30 \mathrm{~min}$. Mouse IgG (Life Technologies, 08-6599) or mouse IgG2ak (BD, 555573) were used as a control for $\mathrm{mAb} 1 \mathrm{C} 01$, rabbit polyclonal IgG (ab27472) was the control for rabbit polyclonal anti-NKp30 (Abnova, PAB17794).

Single-cell force spectroscopy. Experiments to determine binding forces using Atomic Force Microscopy (AFM)-based SCFS were performed using a temperature-controlled incubator at $37^{\circ} \mathrm{C}$, supplemented with $5 \% \mathrm{CO}_{2}$. A JPK Cellhesion unit (JPK, Berlin, Germany) was used to determine the binding forces. A glass was coated with $0.01 \%$ poly-L-lysine and $\beta$-1,3-glucan or mannan was loaded into the chambers and air dried. Depending on the experiment, a single YT cell or one to six polystyrene beads were attached to a cantilever tip $(320 \mu \mathrm{m}$ long and $22 \mu \mathrm{m}$ wide with spring constant of $0.03 \mathrm{~N} / \mathrm{m}$ ) with Cell-Tak (Corning, \#354240). Each cantilever with cells or beads was calibrated against the blank control glass dish surface to analyze its thermal fluctuation. All measurements were performed using JPK NanoWizard II with the Cellhesion module (JPK Instruments AG). Briefly, the cantilever was lowered onto a glass dish containing a thin layer of Cell-Tak. The cantilever was moved away from the Cell-Tak while touching the dish surface to wipe off the majority of the Cell-Tak, but retaining enough to glue a YT cell or beads to the tip. The cantilever was then moved to a different dish and gently lowered until the beads or cells were touching the Cell-Tak and fixed on the tip. The recombinant ectodomain of NKp30, $\beta$-1,3-glucan, or mannan was coated onto a poly-L-lysine-coated dish. The YT cell or bead(s) on the tip were submerged in $1 \mathrm{ml}$ complete RPMI in the SCFS incubator throughout the experiment. The cantilever tip with the attached cell or bead(s) was lowered toward, touched, and lifted off the $\beta$-1,3-glucan or NKp30-coated glass surface (IP gain: $5 \mathrm{~Hz}$; IG gain: $0.0002 \mathrm{~Hz}$; correct baseline: 1 ; relative set point: $0.16-0.5 \mathrm{nN} ; z$ length: $10 \mu \mathrm{m}$; extend time: $3 \mathrm{~s}$; extend delay: $3-7 \mathrm{~s}$; constant height, extend/retract speed: 10 or 5 $\mu \mathrm{m} / \mathrm{s}$, respectively) 20-30 times. Retraction force between the single YT cell and the $\beta$-1,3-glucan surface or fixed bead(s) on the cantilever and NKp30-coated surface on the dishes was recorded. Measurements were repeated on more than three different areas of the coated glass dish. Representative F-D curves were used to show the retraction force vs. distance. Retraction force was the force used to detach the cell or beads on the cantilever tip from the coating on the glass dish, and distance was the distance between the tip and the coating on the dish. F-D curves were normalized and analyzed using JPK Data Processing software (JPK Instruments $A G)$.

Fluorescence microscopy and live cell imaging. For fluorescent microscopy, $1 \mathrm{C} 01$ was used to label NKp30 for immunocytochemistry and Lysotracker Red DND-99 (ThermoFisher, L7528) was used to label granules for live cell imaging. Briefly, YT cells were incubated with $5 \mu \mathrm{l} \mathrm{mAb} 1 \mathrm{C} 01(2.5 \mu \mathrm{g} / \mathrm{ml})$ on ice for $30 \mathrm{~min}$ and the labeling was visualized using a goat anti-mouse antibody conjugated with Alexa 555 (red, Molecular Probes, A-21422). To block NKp30 and assess granule (Lysotracker positive) movement, YT cells were pretreated with $25 \mu \mathrm{l} 1 \mathrm{C} 01$ or an equivalent amount of isotype $\operatorname{IgG}$ at $37^{\circ} \mathrm{C}$ for $30 \mathrm{~min}$ before polystyrene beads (stimulus or control) were added and co-cultured at $37^{\circ} \mathrm{C}$ for the entire period of experiment. The cells were mounted onto glass slides, fixed, and labeled with secondary antibody against 1C01, anti-perforin-FITC antibody (BD 556577, green), and DAPI. Conjugate formation was determined using differential interference contrast (DIC) imaging. Both DIC and fluorescent imaging were performed using Delta Vision microscopy (Applied Precision Inc.) with a PlanApo $\times 60$ objective (1.42 NA) equipped with stacking capabilities. DIC and fluorescence images represented one de-convolved Z-stack obtained using the digital deconvolution program, SoftWoRx v3.5.1, API (Issaquat, WA, USA). Representative images were contrast-enhanced for clarity using SoftWoRx v3.5.1.
Total internal reflection fluorescence microscopy. For TIRF imaging, a Leica DMI6000 B inverted microscope was used, which has adaptive focus control, multi-modal optical head, and fast spinning disk (SD) confocal capacity. An 8chamber dish was pre-coated with $0.01 \%$ poly-L-lysine, and $\beta$-1,3-glucan or mannan was loaded into the chambers and air dried. YT cells were then loaded into the dish chambers and incubated at $37^{\circ} \mathrm{C}$ for $4 \mathrm{~h}$. The cells were fixed with $1 \%$ formaldehyde and stained with $1 \mathrm{C} 01$, and the labeling was visualized with a goat anti-mouse secondary antibody conjugated with Alexa 555 (Invitrogen). To capture the labeling only at the IS at the cell surface, a depth of $80-100 \mathrm{~nm}$ from the lens was set and imaged at high magnification ( $\times 63$, oil immersion). SD microscopy was used to visualize NKp30 labeling throughout the YT cell body, including the IS. Mannan and poly-L-lysine coating alone served as controls.

Imaging quantifications. Volocity image analysis software (Perkin Elmer, Waltham, MA) was used to analyze the movies and quantify the Lysotracker-stained granules trafficking and determine distances to IS. ImageJ (NIH) was used to quantify the NKp30 staining intensities in TIRF microscopy, and a program was written to quantify the distribution of NKp30 and perforin in cells, the location of fluorescent pixels in relation to the IS are described. The point of contact of YT cell with the bead was manually established and set as a reference point to indicate the synapse. The "find spot" measurement function was used to identify granules. Background staining was excluded by adjusting spot intensity to identify only fluorescent granules. For measurements of granule distances, spot intensity was manually offset by $50 \%$ with the brightest spot within a radius of $0.5 \mu \mathrm{m}$. Distances of the granules to the synapse were determined by measuring the distance from the centroid of each granule in the YT cell to the point of contact with the bead over time.

For Image (NIH) quantification, a quadrilateral was drawn over the edges of each cell encompassing the whole cell body in the images and gray values of each pixel in the quadrilateral were measured by the plotting profile function in ImageJ as illustrated (Supplementary Fig. 6A). The gray values of each cell in the images were measured and a graphic figure (Supplementary Fig. 6B) was created with the vertical axis as gray values in arbitrary units (au). All the values from all the cells were compiled to generate the figures (Fig. $5 \mathrm{~g}$ ).

To quantify the distribution of NKp30 and perforin in cells, the location of fluorescent pixels in relation to the IS were measured by calculating the angles of each pixel in relation to the radius from the center of the cell to the center of the IS as illustrated (Supplementary Fig. 6C). The IS was determined manually as the mid-point of the interface between YT cells and beads. The center of the cell was determined manually. Another line was drawn between pixels with fluorescence above a threshold of $0.2(\mathrm{NKp} 30)$ or 0.4 (perforin) corresponding to $10 \%$ of the full dynamic range of the 12-bit camera and the center of the cell. An illustration in Supplementary Fig. 6C showing an angle $\alpha<45^{\circ}$ or $\beta$, greater than $45^{\circ}$ between these two lines was determined for each pixel. For the analysis, a program written in R language ${ }^{62}$ using RStudio ${ }^{63}$ with libraries from EBImage ${ }^{64}$ was used to identify each fluorescent pixel in each image. The number of the pixels above a set threshold was plotted against their respective angles from the IS (histograms). NKp30 clustering or perforin polarization was defined when the peak of positive pixels was at an angle of $<45^{\circ}$ to the IS.

Statistics. Statistical studies were performed using GraphPad Prism (GraphPad Software, Inc. La Jolla, USA). One-way ANOVA with Bonferroni comparison tests with Welch correction or unpaired T-test (two-tailed) was used to evaluate differences among conditions with error bars of SEM unless otherwise specified. In all cases $p<0.05$ was considered significant.

Study approval. All recruited volunteers provided written informed consent. Use of human materials was approved by The Conjoint Health Research Ethics Board, and all experiments including samples from HIV-infected patients were performed in a BSL2+laboratory.

Data availability. The authors declare that the data supporting the findings of this study are available within the article and its supplementary information files, or are available upon reasonable requests to the authors.

Received: 25 April 2017 Accepted: 11 January 2018 Published online: 21 February 2018

\section{References}

1. Vallabhaneni, S., Mody, R. K., Walker, T. \& Chiller, T. The global burden of fungal diseases. Infect. Dis. Clin. North. Am. 30, 1-11 (2016).

2. Enoch, D. A., Yang, H., Aliyu, S. H. \& Micallef, C. The changing epidemiology of invasive fungal infections. Methods Mol. Biol. 1508, 17-65 (2017).

3. Kullberg, B. J. \& Arendrup, M. C. Invasive Candidiasis. N. Engl. J. Med. 373 1445-1456 (2015). 
4. Jarvis, J. N. et al. Determinants of mortality in a combined cohort of 501 patients with HIV-associated Cryptococcal meningitis: implications for improving outcomes. Clin. Infect. Dis. 58, 736-745 (2014).

5. Hidore, M. R., Mislan, T. W. \& Murphy, J. W. Responses of murine natural killer cells to binding of the fungal target Cryptococcus neoformans. Infect. Immun. 59, 1489-1499 (1991).

6. Hidore, M. R. \& Murphy, J. W. Natural cellular resistance of beige mice against Cryptococcus neoformans. J. Immunol. 137, 3624-3631 (1986).

7. Hidore, M. R. \& Murphy, J. W. Correlation of natural killer cell activity and clearance of Cryptococcus neoformans from mice after adoptive transfer of splenic nylon wool-nonadherent cells. Infect. Immun. 51, 547-555 (1986).

8. Hidore, M. R. \& Murphy, J. W. Murine natural killer cell interactions with a fungal target, Cryptococcus neoformans. Infect. Immun. 57, 1990-1997 (1989).

9. Hidore, M. R., Nabavi, N., Sonleitner, F. \& Murphy, J. W. Murine natural killer cells are fungicidal to Cryptococcus neoformans. Infect. Immun. 59, 1747-1754 (1991).

10. Murphy, J. W., Hidore, M. R. \& Wong, S. C. Direct interactions of human lymphocytes with the yeast-like organism, Cryptococcus neoformans. J. Clin. Invest. 91, 1553-1566 (1993).

11. Levitz, S. M., Dupont, M. P. \& Smail, E. H. Direct activity of human T lymphocytes and natural killer cells against Cryptococcus neoformans. Infect. Immun. 62, 194-202 (1994).

12. Gonzalez-Amaro, R. et al. Natural killer cell-mediated cytotoxicity in cryptococcal meningitis. Rev. Invest. Clin. 43, 133-138 (1991).

13. Horn, C. A. \& Washburn, R. G. Anticryptococcal activity of NK cell-enriched peripheral blood lymphocytes from human immunodeficiency virus-infected subjects: responses to interleukin-2, interferon-gamma, and interleukin-12. J. Infect. Dis. 172, 1023-1027 (1995).

14. Islam, A. et al. An acidic microenvironment increases NK cell killing of Cryptococcus neoformans and Cryptococcus gattii by enhancing perforin degranulation. PLoS Pathog. 9, e1003439 (2013).

15. Li, S. S. et al. The NK receptor NKp30 mediates direct fungal recognition and killing and is diminished in NK cells from HIV-infected patients. Cell Host Microbe 14, 387-397 (2013).

16. Brown, G. D. Dectin-1: a signalling non-TLR pattern-recognition receptor. Nat. Rev. Immunol. 6, 33-43 (2006).

17. Elomaa, O. et al. Cloning of a novel bacteria-binding receptor structurally related to scavenger receptors and expressed in a subset of macrophages. Cell 80, 603-609 (1995).

18. Bowdish, D. M. \& Gordon, S. Conserved domains of the class A scavenger receptors: evolution and function. Immunol. Rev. 227, 19-31 (2009).

19. Ezekowitz, R. A., Sastry, K., Bailly, P. \& Warner, A. Molecular characterization of the human macrophage mannose receptor: demonstration of multiple carbohydrate recognition-like domains and phagocytosis of yeasts in Cos-1 cells. J. Exp. Med. 172, 1785-1794 (1990).

20. Kawai, T. \& Akira, S. The roles of TLRs, RLRs and NLRs in pathogen recognition. Int. Immunol. 21, 317-337 (2009).

21. Vitenshtein, A. et al. NK cell recognition of candida glabrata through binding of NKp46 and NCR1 to fungal ligands Epa1, Epa6, and Epa7. Cell Host Microbe 20, 527-534 (2016).

22. Brueggemann, S., Schmitt, A. L., Einsele, H., Loeffler, J. Characterization of human natural killer cell receptors recognizing Aspergillus fumigatus. In 6 th Advances Against Aspergillosis Madrid, Spain (2014).

23. Mavoungou, E., Held, J., Mewono, L. \& Kremsner, P. G. A Duffy binding-like domain is involved in the NKp30-mediated recognition of Plasmodium falciparum-parasitized erythrocytes by natural killer cells. J. Infect. Dis. 195 , 1521-1531 (2007).

24. Janeway, C. A. Jr. Approaching the asymptote? Evolution and revolution in immunology. Cold Spring Harb. Symp. Quant. Biol. 54(Pt 1), 1-13 (1989).

25. McFadden, D., Zaragoza, O. \& Casadevall, A. The capsular dynamics of Cryptococcus neoformans. Trends Microbiol. 14, 497-505 (2006).

26. de Souza Pereira, R. \& Geibel, J. Direct observation of oxidative stress on the cell wall of Saccharomyces cerevisiae strains with atomic force microscopy. Mol. Cell. Biochem. 201, 17-24 (1999).

27. Nimrichter, L., Rodrigues, M. L., Rodrigues, E. G. \& Travassos, L. R. The multitude of targets for the immune system and drug therapy in the fungal cell wall. Microbes Infect. 7, 789-798 (2005).

28. Sipiczki, M. Where does fission yeast sit on the tree of life? Genome Biol. 1, REVIEWS1011 (2000).

29. Klutts, J. S., Yoneda, A., Reilly, M. C., Bose, I. \& Doering, T. L. Glycosyltransferases and their products: cryptococcal variations on fungal themes. FEMS Yeast Res. 6, 499-512 (2006).

30. Free, S. J. Fungal cell wall organization and biosynthesis. Adv. Genet. 81, 33-82 (2013).

31. Mody, C. H. et al. Proteins in the cell wall and membrane of Cryptococcus neoformans stimulate lymphocytes from both adults and fetal cord blood to proliferate. Infect. Immun. 64, 4811-4819 (1996).
32. Nisizawa, K., Yamaguchi, T., Handa, N., Maeda, M. \& Yamazaki, H. Chemical nature of a uronic acid-containing polysaccharide in the peritrophic membrane of the silkworm. J. Biochem. 54, 419-426 (1963).

33. Tam, J. M., Mansour, M. K., Khan, N. S., Yoder, N. C. \& Vyas, J. M. Use of fungal derived polysaccharide-conjugated particles to probe dectin-1 responses in innate immunity. Integr. Biol. 4, 220-227 (2012).

34. Deresinski, S. C. \& Stevens, D. A. Caspofungin. Clin. Infect. Dis. 36, 1445-1457 (2003).

35. Maligie, M. A. \& Selitrennikoff, C. P. Cryptococcus neoformans resistance to echinocandins: $(1,3)$ beta-glucan synthase activity is sensitive to echinocandins. Antimicrob. Agents Chemother. 49, 2851-2856 (2005).

36. Kyei, S. K., et al. Mechanisms by which Interleukin-12 corrects defective NK cell anticryptococcal activity in HIV-infected patients. MBio 7, e00878-16 (2016).

37. Whited, A. M. \& Park, P. S. Atomic force microscopy: a multifaceted tool to study membrane proteins and their interactions with ligands. Biochim. Biophys. Acta 1838, 56-68 (2014).

38. Zhang, X., Wojcikiewicz, E. \& Moy, V. T. Force spectroscopy of the leukocyte function-associated antigen-1/intercellular adhesion molecule-1 interaction. Biophys. J. 83, 2270-2279 (2002).

39. Zhang, X., Wojcikiewicz, E. P. \& Moy, V. T. Dynamic adhesion of T lymphocytes to endothelial cells revealed by atomic force microscopy. Exp. Biol. Med. 231, 1306-1312 (2006).

40. Tsoni, S. V. \& Brown, G. D. beta-Glucans and dectin-1. Ann. NY Acad. Sci. 1143, 45-60 (2008).

41. Marr, K. J. et al. Cryptococcus neoformans directly stimulates perforin production and rearms NK cells for enhanced anticryptococcal microbicidal activity. Infect. Immun. 77, 2436-2446 (2009).

42. Oykhman, P. et al. Requirement and redundancy of the Src family kinases Fyn and Lyn in perforin-dependent killing of Cryptococcus neoformans by NK cells. Infect. Immun. 81, 3912-3922 (2013).

43. Gratacap, M. P. et al. The new tyrosine-kinase inhibitor and anticancer drug dasatinib reversibly affects platelet activation in vitro and in vivo. Blood 114 1884-1892 (2009).

44. Di Luzio, N. R. \& Williams, D. L. Protective effect of glucan against systemic Staphylococcus aureus septicemia in normal and leukemic mice. Infect. Immun. 20, 804-810 (1978).

45. Reynolds, J. A. et al. Glucan-induced enhancement of host resistance to selected infectious diseases. Infect. Immun. 30, 51-57 (1980).

46. Song, M. \& di Luzio, N. R. Yeast glucan and immunotherapy of infectious diseases. Front. Biol. 48, 533-547 (1979).

47. Williams, D. L. \& Di Luzio, N. R. Glucan-induced modification of murine viral hepatitis. Science 208, 67-69 (1980).

48. Williams, D. L., Cook, J. A., Hoffmann, E. O. \& Di Luzio, N. R. Protective effect of glucan in experimentally induced candidiasis. J. Reticuloendothel. Soc. 23, 479-490 (1978).

49. Cook, J. A., Holbrook, T. W. \& Parker, B. W. Visceral leishmaniasis in mice: protective effect of glucan. J. Reticuloendothel. Soc. 27, 567-573 (1980).

50. Babineau, T. J. et al. Randomized phase I/II trial of a macrophage-specific immunomodulator (PGG-glucan) in high-risk surgical patients. Ann. Surg. 220, 601-609 (1994).

51. Babineau, T. J. et al. A phase II multicenter, double-blind, randomized, placebo-controlled study of three dosages of an immunomodulator (PGGglucan) in high-risk surgical patients. Arch. Surg. 129, 1204-1210 (1994).

52. Dellinger, E. P. et al. Effect of PGG-glucan on the rate of serious postoperative infection or death observed after high-risk gastrointestinal operations. Betafectin Gastrointestinal Study Group. Arch. Surg. 134, 977-983 (1999).

53. Willment, J. A. et al. The human beta-glucan receptor is widely expressed and functionally equivalent to murine Dectin-1 on primary cells. Eur. J. Immunol. 35, 1539-1547 (2005).

54. Taylor, P. R. et al. The beta-glucan receptor, dectin-1, is predominantly expressed on the surface of cells of the monocyte/macrophage and neutrophil lineages. J. Immunol. 169, 3876-3882 (2002).

55. Di Renzo, L., Yefenof, E. \& Klein, E. The function of human NK cells is enhanced by beta-glucan, a ligand of CR3 (CD11b/CD18). Eur. J. Immunol. 21, 1755-1758 (1991).

56. Jones, G. J. et al. In contrast to anti-tumor activity, YT cell and primary NK cell cytotoxicity for Cryptococcus neoformans bypasses LFA-1. Int. Immunol. 21, 423-432 (2009).

57. Ostrosky-Zeichner, L. et al. Multicenter clinical evaluation of the (1-->3) betaD-glucan assay as an aid to diagnosis of fungal infections in humans. Clin. Infect. Dis. 41, 654-659 (2005).

58. Miyazaki, T. et al. Plasma (1-->3)-beta-D-glucan and fungal antigenemia in patients with candidemia, aspergillosis, and cryptococcosis. J. Clin. Microbiol. 33, 3115-3118 (1995).

59. Denning, D. W. Echinocandin antifungal drugs. Lancet 362, 1142-1151 (2003). 
60. Krensky, A. M. \& Clayberger, C. Biology and clinical relevance of granulysin. Tissue Antigens 73, 193-198 (2009).

61. Li, Y., Wang, Q. \& Mariuzza, R. A. Structure of the human activating natural cytotoxicity receptor NKp30 bound to its tumor cell ligand B7-H6. J. Exp. Med. 208, 703-714 (2011)

62. Team RDC. R: A Language and Environment for Statistical Computing (The R Foundation for Statistical Computing, Vienna, 2011).

63. RStudio. RStudio: Integrated Development Environment for $R$ (Version 0.98, 1091) (RStudio, Inc, Boston, 2012)

64. Pau, G., Fuchs, F., Sklyar, O., Boutros, M. \& Huber, W. EBImage--an R package for image processing with applications to cellular phenotypes. Bioinformatics 26, 979-981 (2010).

\section{Acknowledgements}

This work was supported by a grant from the Canadian Institutes for Health Research (CIHR) (CHM \#365812), the Jessie Bowden Lloyd Professorship in Immunology (CHM), by an equipment and infrastructure grant from the Canadian Foundation for Innovation (CFI), the Alberta Science and Research Authority, and the Jessie Bowden Lloyd Professorship (S.S.L.). Work with immunofluorescence microscopy and Leica DMI6000 B Inverted research level microscope were supported by the Live Cell Imaging Facility, funded by the Snyder Institute for Chronic Disease, University of Calgary, with invaluable assistance from Dr. Pina Colarusso and Ms. Jennifer (Amon) Poirier. The authors extend thanks to Dr. M. John Gill who provided blood samples from HIVinfected patients and to Danuta Stack and Martina Timm-McCann for their expert technical assistance.

\section{Author contributions}

S.S.L. conceived the concepts, designed the overall study, planned and executed experiments, performed data analyses, and wrote the manuscript; H.O. performed the microscope imaging and analysis; M.K.M. and J.M.V prepared the polystyrene beads conjugated to $\beta$-1,3-glucan or mannan. R.X. did the signaling studies, wrote the software, and analyzed staining distribution; L.S. performed part of the fungal killing assay; R.M. created the recombinant ectodomain NKp30 protein; F.M., P.M. and M.A. provided expert support on AFM; S.M.R. provided intellectual advice and review of the manuscript; and C.H.M. provided intellectual input, discussed results, and edited the manuscript. All authors reviewed the manuscript.

\section{Additional information}

Supplementary Information accompanies this paper at https://doi.org/10.1038/s41467018-03014-4.

Competing interests: The authors declare no competing financial interests.

Reprints and permission information is available online at http://npg.nature.com/ reprintsandpermissions/

Publisher's note: Springer Nature remains neutral with regard to jurisdictional claims in published maps and institutional affiliations.

\section{(c) (i)}

Open Access This article is licensed under a Creative Commons Attribution 4.0 International License, which permits use, sharing, adaptation, distribution and reproduction in any medium or format, as long as you give appropriate credit to the original author(s) and the source, provide a link to the Creative Commons license, and indicate if changes were made. The images or other third party material in this article are included in the article's Creative Commons license, unless indicated otherwise in a credit line to the material. If material is not included in the article's Creative Commons license and your intended use is not permitted by statutory regulation or exceeds the permitted use, you will need to obtain permission directly from the copyright holder. To view a copy of this license, visit http://creativecommons.org/ licenses/by/4.0/.

(C) The Author(s) 2018 